\title{
Imaging Artifacts of Medical Instruments in Ultrasound-Guided Interventions
}

\author{
Jinlan Huang, PhD, John K. Triedman, MD, \\ Nikolay V. Vasilyev, MD, Yoshihiro Suematsu, MD, PhD, \\ Robin O. Cleveland, PhD, Pierre E. Dupont, PhD
}

\begin{abstract}
Objective. Real-time 3-dimensional (3D) ultrasound imaging has the potential to become a dominant imaging technique for minimally invasive surgery. One barrier to its widespread use is that surgical instruments generate imaging artifacts, which can obfuscate their location, orientation, and geometry and obscure nearby tissue. The purpose of this study was to identify and describe the types of artifacts which could be produced by metallic instruments during interventions guided by 3D ultrasound imaging. Methods. Three imaging studies were performed. First, imaging artifacts from stainless steel rods were identified in vitro and acoustically characterized. Second, 3 typical minimally invasive instruments were imaged (in vitro and in vivo), and their artifacts were analyzed. The third study compared the intensity of imaging artifacts (in vitro and in vivo) from stainless steel rods with rods composed of 3 different materials and stainless steel rods with roughened and coated surfaces. Results. For the stainless steel rods, all observed artifacts are described and illustrated, and their physical origins are explained. Artifacts from the 3 minimally invasive instruments are characterized with the use of the artifacts observed with the rods. Finally, it is shown that artifacts can be greatly reduced through the use of alternate materials or by surface modification. Conclusions. Instrument artifacts in 3D ultrasound images can be more confusing than those from the same instruments imaged in 2 dimensions. Realtime 3D ultrasound imaging can, however, be used effectively for in vivo imaging of minimally invasive instruments by using artifact mitigation techniques, including careful selection of probe and incision locations, as well as by instrument modification. Key words: imaging artifacts; medical instruments; ultrasound-guided interventions.
\end{abstract}

\begin{abstract}
Abbreviations
3D, 3-dimensional; 2D, 2-dimensional

Received March 12, 2007, from the Department of Cardiology, Division of Basic Cardiovascular Research, Children's Hospital Boston, Boston, Massachusetts USA (J.H., J.K.T., N.V.V.); Department of Cardiothoracic Surgery, University of Tokyo, Tokyo, Japan (Y.S.); and Department of Aerospace and Mechanical Engineering, Boston University, Boston, Massachusetts USA (R.O.C., P.E.D.). Revision requested April 13, 2007. Revised manuscript accepted for publication May 30, 2007.

This work was supported by National Institutes of Health National Institute of Biomedical Imaging and Bioengineering grant 1 RO1 EB003052 and National Institutes of Health Bioengineering Research Partnership grant R01 HL073647.

Address correspondence to Pierre E. Dupont, PhD, Department of Aerospace and Mechanical Engineering, Boston University, 110 Cummington St, Boston, MA 02215 USA.

E-mail: pierre@bu.edu
\end{abstract}

Video online at www.jultrasoundmed.org

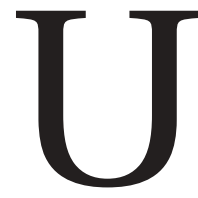

ltrasound imaging has enjoyed widespread use for diagnostics in almost every branch of medicine, owing to its real-time capability, low cost, and avoidance of ionizing radiation. It has also been growing in popularity for guiding interventional procedures because it enables continuous and simultaneous visualization of tissue and the surgical instrument during the procedure. ${ }^{1-3}$ The recent introduction of commercial real-time 3-dimensional (3D) imaging systems is likely to facilitate ultrasound guidance of more complex interventional procedures. ${ }^{4-6}$ One example under study by the authors is beating heart intracardiac surgery. ${ }^{7-9}$

A current barrier to the interventional use of ultrasound is the difficulty encountered in visualizing metal instruments. Often the instrument either is not visible or induces strong artifacts that obscure the instrument and surrounding tissue. Figure 1 depicts an example in which a rod's tip is positioned at the center of an in vitro pul- 
satile model of the mitral valve constructed from porcine mitral valve tissue. The reader's viewpoint of Figure 1A (depicted as if imaging the actual valve) was used to generate the $3 \mathrm{D}$ ultrasound image in Figure 1B. At this angle of insonification, only the rod's tip is clearly visible. Furthermore, artifacts generated by the rod result in an enlarged tip and a fictional object appearing below the rod, which overlaps the mitral annulus.

Imaging artifacts arise when the signal-processing assumptions made by the ultrasound imaging system are violated. The major assumptions include the following: (1) the acoustic waves travel in straight lines; (2) the waves are infinitely thin in their lateral extent; (3) each interface generates a single echo or reflection; (4) the intensity of returning echoes is directly related to the scattering strength of the imaged objects; (5) sound speed and attenuation are homogeneous and known a priori; and (6) any detected echo is due to the most recently transmitted acoustic pulse. Mild violations of these assumptions do occur in soft tissue, and the concomitant artifacts have been described extensively. ${ }^{10-15}$ In the case of metallic instruments (eg, stainless steel), the violations are more egregious: the sound speed is different from that of tissue by a factor of 3 to 4 , and the acoustic impedance is different by a factor of up to 10 . This problem is compounded by the specular nature of reflections arising from the instruments' typically smooth surfaces. The result is that, when imaging an instrument, the ultrasound system receives a very strong echo at normal incidence, which can saturate the image, and almost no signal at oblique incidence, in which case the surface becomes invisible.

Most instrument artifacts fall into 2 categories: those arising from the reverberation of sound within an instrument and those arising from echoes generated within the side lobes of the ultrasound beam. These 2 categories are described below along with a number of additional artifacts.

\section{Reverberation Artifacts}

Reverberation refers to the multiple echoes that can occur when sound is reflected repeatedly inside an object or between 2 objects, 1 of which can be the probe. The simplest example corresponds to an object with flat, parallel surfaces positioned orthogonal to a scan line. For instru- ments, the reverberation process is more complex because instruments rarely have flat parallel surfaces and, furthermore, they are elastic solids that can support both compression and shear

Figure 1. Three-dimensional ultrasound image of a rod inserted into an in vitro pulsatile model of the porcine mitral valve. A, Schematic indicating relative probe and rod locations relative to actual anatomy. B, Image of in vitro model depicted from the reader's viewpoint of $\mathbf{A}$ showing artifacts.

\section{A}

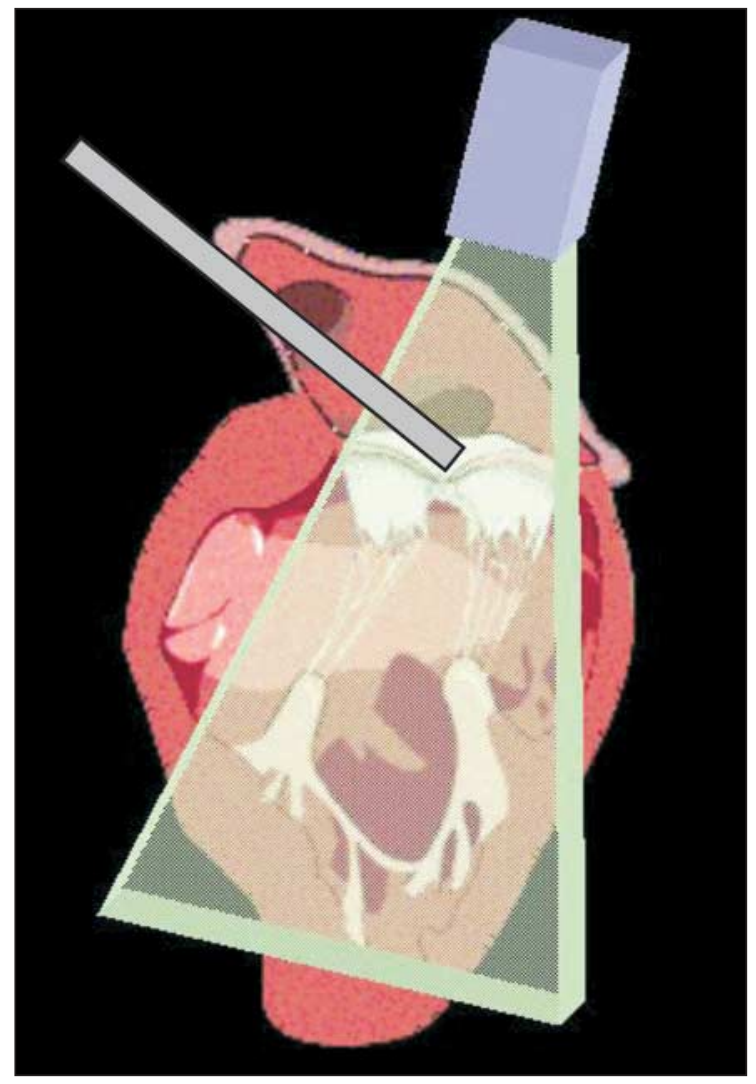

B

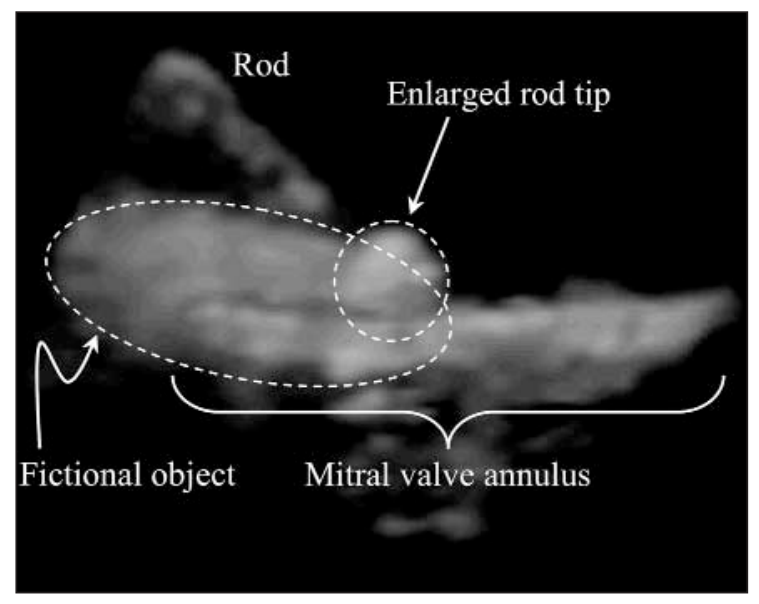


waves. The transmission of ultrasound into an instrument can excite various modes of these waves, which can reverberate and return multiple echoes to the transducer. Even a relatively simple geometric shape such as a cylinder can result in a rich backscatter structure, generating compressional waves, shear waves, interface/surface waves, and creeping or circumferential waves. ${ }^{16-20}$ The resulting artifact on the image is referred to as a comet tail, and the earliest reports described images of shotgun pellets in human tissue..$^{21,22}$ The comet tail artifact has also been observed at tissue-gas interfaces, within small calcified structures, and in the presence of foreign bodies. ${ }^{23-26}$

We note that an artifact that closely resembles the appearance of the comet tail artifact is the ring-down artifact. ${ }^{27-29}$ The ring-down artifact arises from the resonance associated with air cavities in response to an ultrasound pulse. It is reported that a horn- or bugle-shaped fluid collection entrapped between bubbles is needed to produce the appropriate resonant structure to result in a ring-down artifact. ${ }^{27}$ This artifact could potentially occur if bubbles are trapped on the surface of instruments when they are inserted into the body.

Instruments can also give rise to guided wave reverberations. Because the shaft of an instrument can act as a waveguide, insonification at non-normal incidence can cause a portion of the incident energy to be converted into modes that propagate along the shaft of the instrument. Cylindrical rods can support many possible waveguide modes. ${ }^{30,31}$ These modes can reflect repeatedly from discontinuities in the shaft and leak out of the instrument as acoustic waves that can be detected by the transducer.

\section{Side Lobe Artifacts}

One of the assumptions used by ultrasound imaging systems is that the lateral width of the ultrasound beam is infinitely thin, but diffraction prevents this from being realized in practice. Most ultrasound beams employ weak focusing, which results in a relatively long collimated beam with a small, but finite, lateral extent. The $-6-\mathrm{dB}$ beamwidth is on the order of a wavelength, but there is an acoustic signal well beyond the nominal beam width, albeit at very low amplitude. For a continuous wave source, the diffraction in the focal plane is described by a sinc function, which has a well-defined main lobe followed by a series of side lobes. ${ }^{32}$ For the short pulses used in ultrasound imaging, the "side lobes" are smeared out and no longer consist of clear peaks and nulls. Furthermore, we note that for phased arrays, the side lobes result from both the directivity of the individual elements and the directivity of the array (the spacing and length of the array). In cases in which the spacing between elements of the array (the pitch) is greater than a wavelength, then constructive interference between the elements in the array results in "grating lobes" 32 or "secondary major lobes," 33 in which the amplitude of the lobe is comparable with that of the main beam. This is not desirable for imaging applications, and in most ultrasound systems, the pitch between elements is less than half a wavelength, so grating lobes are usually not present. In what follows, the term side lobes is used to describe any acoustic energy that is outside the main beam because of diffraction effects.

For soft tissue, the scattering strength is relatively uniform, and the side lobe levels are typically better than $-40 \mathrm{~dB}$. The resulting echoes have a negligible impact in comparison with the signals from the main lobe. If a strong scatterer is present in the side lobe, however, it can produce an echo that is not negligible. The processing carried out by the imaging system assumes that all signals originate from the main lobe, and the signal from the scatterer will be displayed as an object in the direction of the main beam. Transducer side lobes can create both low-level diffusive echoes and bright specular reflections in certain tissue structures. ${ }^{34}$ Specular side lobe artifacts occur near strong, curved, highly reflecting surfaces such as the diaphragm or near large cystic masses such as the urinary bladder and gallbladder. Diffuse side lobe artifacts can originate from bowel gas adjacent to cystic structures.

A number of additional artifacts have been identified in tissue imaging, including speckle, ${ }^{35-37}$ mirror images, ${ }^{38-40}$ shadowing and enhancement, ${ }^{41-44}$ section thickness, ${ }^{45,46}$ refraction, ${ }^{41-43}$ speed error, ${ }^{47,48}$ and range ambiguity. ${ }^{49,50}$ Several of these also appear to be important for instrument imaging as described below. 
The literature on ultrasound imaging of instruments has principally been focused on needles and, in particular, enhancing their visualization by creating a global increase in echogenicity. ${ }^{51-55}$ Most commonly proposed is the addition of an inhomogeneity to an otherwise smooth instrument to improve visualization of the entire shaft at different insonation angles. A recent article of ours reviewed this topic. ${ }^{56}$ Characterization of instrument artifacts in the literature is extremely limited. We are aware of a single study of the use of ultrasound to monitor fetoscopy. ${ }^{57}$ In that study, a curved pattern artifact was observed projecting from the intraamniotic end of a fetoscope.

The goal of this article was to characterize the artifacts that can be observed with metallic instruments used in interventional procedures guided by 3D ultrasound imaging. In the first study, an artifact taxonomy was developed from images of stainless steel rods. Although some of the observed artifacts were similar to those produced in soft tissue, others were specific to instruments. The second study evaluated the artifacts produced by 3 minimally invasive instruments and interpreted them in terms of the results of the first study. The article concludes with a discussion of techniques for reducing the effects of artifacts during interventions and includes a third study, which considered the effects of material choice and surface modification on imaging artifacts.

\section{Materials and Methods}

All images were taken with a clinical echocardiography machine and a $4-\mathrm{MHz}$ probe (SONOS 7500 and X4 probe; Philips Medical Systems, Bothell, WA). The SONOS 7500 system allows 3D imaging with instantaneous online volume-rendered reconstruction as well as direct manipulation of thresholding and cut planes. The transducer operates in a broadband 2- to $4-\mathrm{MHz}$ range and scans a $3 \mathrm{D}$ volume by electronically steering the acoustic beam using a matrix of approximately 3000 transducer elements and associated electronics that allow scanning of a $64^{\circ} \times 64^{\circ}$ pyramidal volume in real time at up to 28 frames per second. The SONOS 7500 base system volume renders the data in any viewing orientation desired, also at a $28-\mathrm{Hz}$ frame rate, and the orientation of the target object on the screen can be controlled with a trackball. Therefore, the operator can view the target from any angle without moving the imaging transducer. The image-processing and -rendering platform supports multiple imaging modalities, including conventional B-mode 2dimensional (2D) echo, 2D color flow Doppler imaging, biplanar 2D echo, and several realtime volume-rendering modes.

In vitro experiments were carried out in a tank (Figure 2) that has been described in detail previously ${ }^{56}$ Briefly, the tank was filled with filtered, deionized, degassed water, and the bottom was lined with silicone rubber to reduce reverberations. The rod or instrument to be visualized was mounted to a rotational stage so that the angle of insonation could be varied. The X4 probe was mounted to a 3 -axis positioning system and was initially translated to ensure that the instrument was placed in the focal plane. The desired orientation of the rod relative to the probe was then obtained by adjusting the rotation stage. The focal length and the imaging depth could be varied as desired. A 2D image was then acquired and saved as a TIFF (tagged image file format) image file. The corresponding 3D images were then acquired and saved as DICOM (Digital Imaging and Communications in Medicine) files. In post analysis, the DICOM files were imported, and 3D volumes could then be viewed from any angle. Once a desired viewing orientation was obtained, an AVI (audiovideo interleave) movie file was saved to disk. A desired frame of the movie file was saved as a TIFF image.

Figure 2. Schematic of the imaging apparatus.

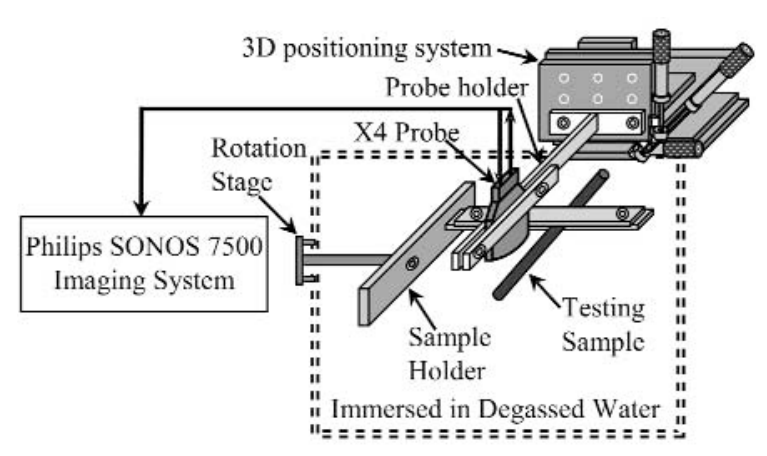

J Ultrasound Med 2007; 26:1303-1322 
In vivo experiments were carried out on male Yorkshire pigs $(70-80 \mathrm{~kg}$ ) with the same imaging system. The experimental protocol was approved by the Children's Hospital Boston Institutional Animal Care and Use Committee. All animals received humane care in accordance with the 1996 Guide for the Care and Use of Laboratory Animals recommended by the US National Institutes of Health. The animal was anesthetized, and a median sternotomy was performed to allow access to the right atrium of the heart. The probe was inserted into a sterile sleeve (CIVCO Medical Instruments, Kalona, IA) filled with an ultrasound gel (Parker Laboratories, Inc, Fairfield, NJ) providing approximately $2 \mathrm{~cm}$ of standoff. The outer surface of the sleeve was moistened with sterile $0.9 \%$ sodium chloride solution and applied to the surface of the right atrium. Two purse string sutures of 3-0 polypropylene were placed on the right atrial appendage for instrument insertion. In the second study, minimally invasive instruments (which will be described below) were inserted into the right atrium of the beating porcine heart, and 3D images were taken to show the artifacts they may produce in vivo. In the third study, stainless steel rods with different surface modifications were inserted into the right atrium of the beating porcine heart, and 3D images were taken to show how surface modifications may improve the visibility of the instruments and reduce artifacts.

Cylinders serve as a convenient geometry for characterizing instrument artifacts because they are accurate representations of an instrument's shaft, and furthermore, many tip-mounted tools can be approximately modeled as a collection of cylinders. Stainless steel (type 304) rods (Figure 3D) were used in the first study as canonical objects with which to identify and characterize instrument imaging artifacts.

The 3 minimally invasive instruments used in the second study are also depicted in Figure 3. Cylindrical needles (Figure 3A) are the most commonly used tools in ultrasound-guided interventions (eg, needle biopsies, drug delivery, and hyperthermia therapy). Figure 3B shows a suturing device consisting of 2 semicylinders, which slide relative to each other along the instrument's axis (developed by Y. Suematsu and
Mani Inc, Tochigi, Japan). There is a concave slot on the tip of the device for the needle. A forceps (Figure 3C) consists of a hollow cylindrical shaft containing a cable to control finger position. The 2 fingers of the forceps are tapered semicylinders with ridged grasping surfaces. These descriptions validate the statement above that instruments can often be modeled as collections of cylinders.

\section{Results}

\section{Artifacts of Cylindrical Rods}

As anticipated, most imaging artifacts produced by stainless steel rods are due to either reverberation or side lobes. Four types of reverberation artifacts and 2 types of side lobe artifacts were observed. These are described below along with 3 additional artifacts.

\section{Reverberation Artifacts}

Comet Tail Artifact-The comet tail artifact is shown for a rod in Figure 4. The bandlike structure is strongest when the rod is exactly perpendicular to the beam (Figure 4, beam 1). For a linear scan, the bandlike structure will be uniform along the length of the instrument. For a sector scan, as used here, the angle of incidence will vary across the length of the instrument because of the steering of the beam. As the angle of incidence departs from normal, the tail becomes weaker because the multiple reflections do not return directly to the transducer (Figure 4, beam 2) until the reflections are direct-

Figure 3. Clinical tool examples. A, 19-gauge needle. B, Suturing device. C, Forceps. D, Stainless steel rod with conical tip.

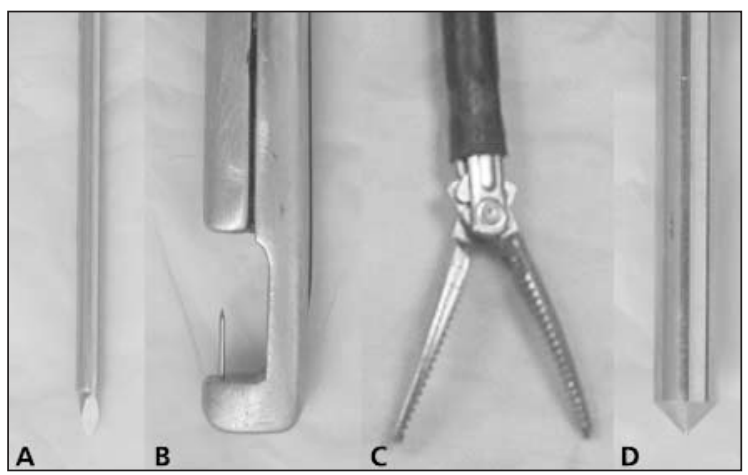




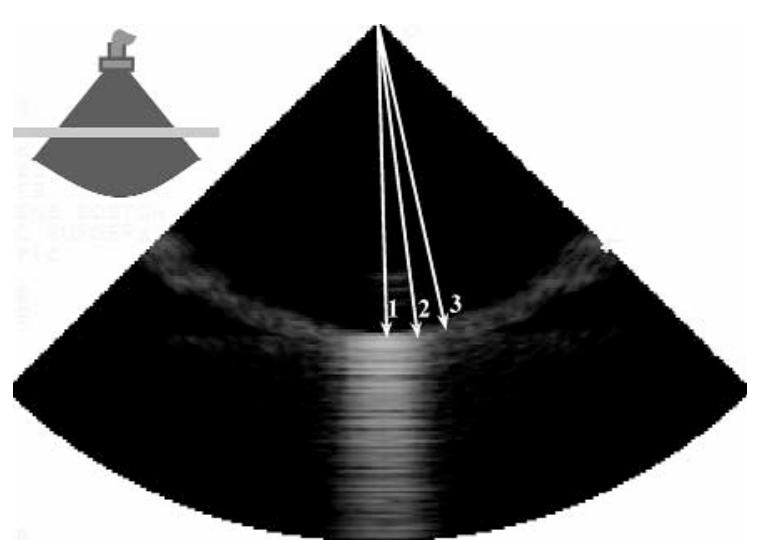

A Both rods are in the scan plane.

ed far enough away that they are not detected by the transducer (Figure 4, beam 3). Indeed, at this angle, the reflection from the surface is not present in the image. The curved hazy region is a side lobe artifact that will be discussed later. In the comet tail, the band structure is quite rich; that is, it does not appear to produce periodic banding. This appears to be because the ultrasound pulse couples into a number of modes in the rod so that each produces echoes back to the imaging transducer.

Guided Wave Artifact-A reverberation that is more specific to an instrument is due to signals that travel along its shaft and reflect from discon-

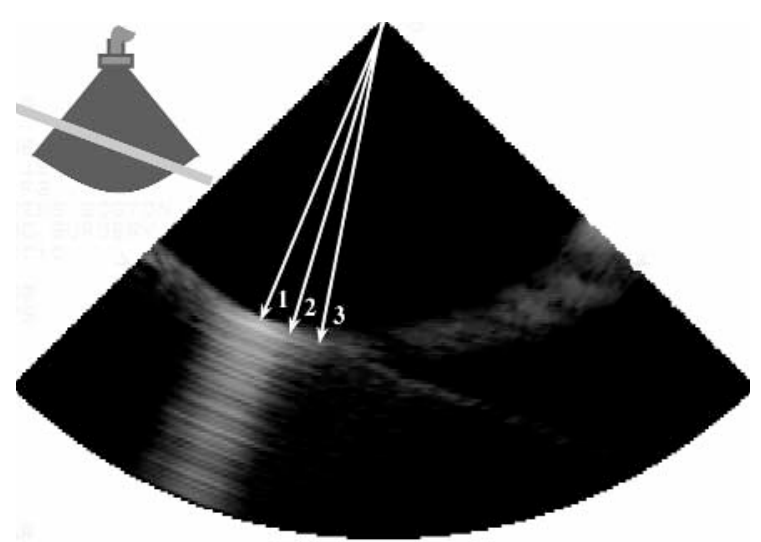

B tinuities along its length. Figure 5 shows an image of a horizontal rod where the tip is on the right side of the image. Although the comet tail artifact can be seen at normal incidence, the guided wave artifacts appear as 3 "fingers" emanating from the tip of the rod, which curve down and to the left.

This artifact is produced when the incident acoustic beam couples into modes that travel along the shaft of the rod. These modes reflect from the end of the rod, or any other discontinuity, such as a hinge, and propagate back to the transducer. Figure 6 demonstrates the acoustic path, which consists of a wave propagating to the instrument, the guided wave that propagates to

Figure 5. A, Image of the guided wave artifact with 3 fingers emanating from the tip. B, Magnified view of fingers with predicted guided wave echo locations for wave speeds of 4500,3300 , and $2300 \mathrm{~m} / \mathrm{s}$ (top to bottom). Axis numbers indicate distance in centimeters.

A

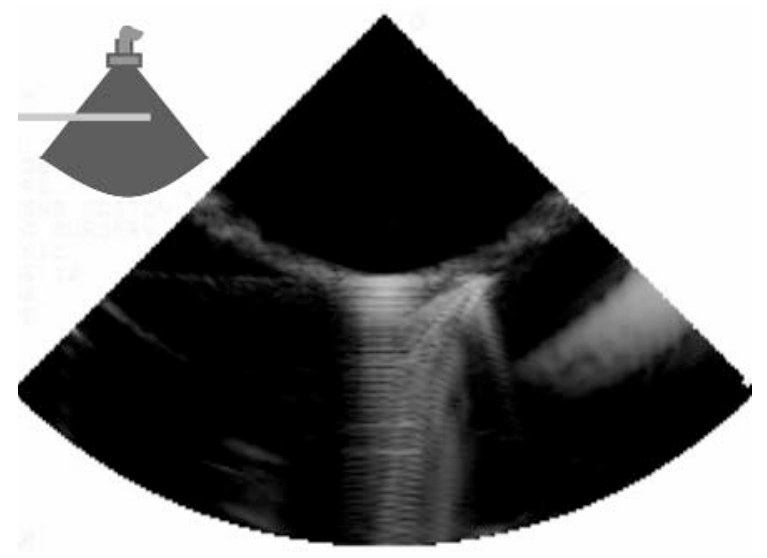

B

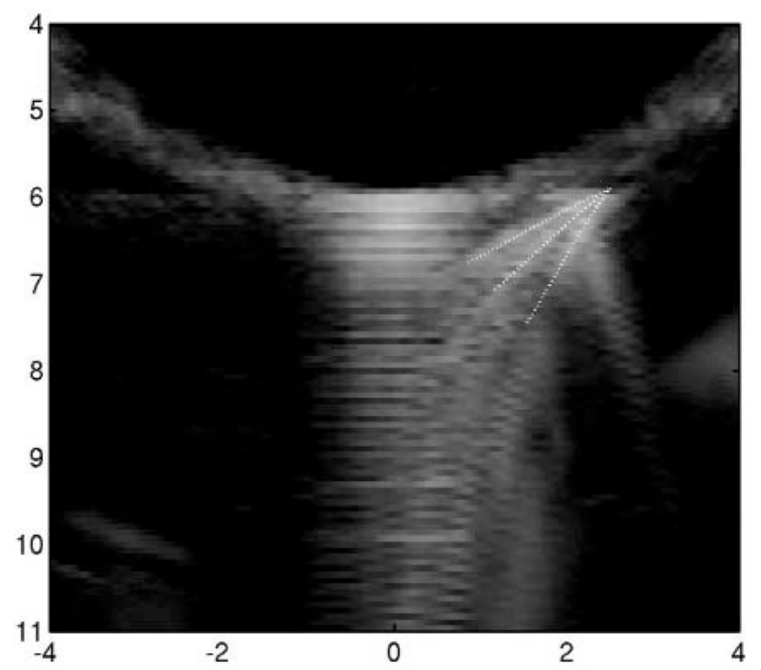




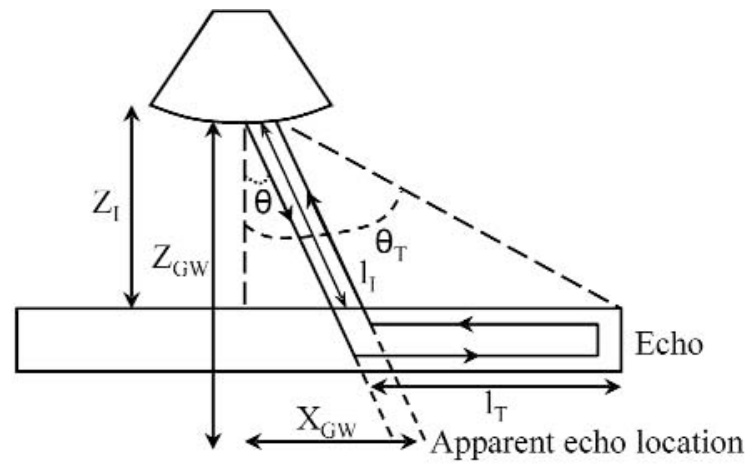

Figure 6. Schematic showing the geometry of the guided wave artifact. The solid lines with arrows show the actual propagation path of the waves. The dashed lines show the propagation path assumed by the imaging system.

the end of the rod and back, and a wave that radiates back toward the transducer. The total travel time for the reflection of the guided wave from the tip of the instrument will be

$$
\text { (1) } t_{\mathrm{GW}}=2 \frac{l_{\mathrm{I}}}{c_{0}}+2 \frac{l_{\mathrm{T}}}{c_{\mathrm{GW}}} \text {, }
$$

where $l_{\mathrm{I}}=z_{\mathrm{I}} / \cos (\theta)$ is the path length to the surface of the instrument; $z_{\mathrm{I}}$ is the perpendicular (vertical) separation between the probe and the instrument; $\theta$ is the angle of the incident beam; $c_{0}$ is the sound speed in tissue; $l_{\mathrm{T}}$ is the length to the end of the instrument; and $c_{\mathrm{GW}}$ is the effective propagation speed of the guided wave along the axis of the instrument. (Note that this speed is governed by the material properties and the geometry of the instrument and the mode that is excited.) The ultrasound imaging system will interpret this echo as coming from a distance $t_{\mathrm{GW}}$ $c_{0} / 2$. In terms of the imaging coordinate system, the apparent horizontal and vertical position of the tip will be

$$
\begin{aligned}
& x_{\mathrm{GW}}=z_{\mathrm{I}}\left[\tan \theta+\frac{c_{\mathrm{o}}}{c_{\mathrm{GW}}}\left(\tan \theta_{\mathrm{T}}-\tan \theta\right) \sin \theta\right] \\
& z_{\mathrm{GW}}=z_{\mathrm{I}}\left[1+\frac{c_{\mathrm{o}}}{c_{\mathrm{GW}}}\left(\tan \theta_{\mathrm{T}}-\tan \theta\right) \cos \theta\right]
\end{aligned}
$$

where $\theta_{\mathrm{T}}$ is the angle to the tip of the instrument. The presence of 3 fingers in Figure 5A indicates that 3 types of guided waves were excited by the ultrasound pulses. Each traveled along the rod, was reflected, and resulted in an echo signal detected by the transducer. Figure 5B overlays on the image the echoes predicted by Equation 2 for 3 different guided wave speeds: 4500, 3300, and $2300 \mathrm{~m} / \mathrm{s}$. The curves provide a good match with the image and support the guided mode hypothesis. Note that these guided wave speeds are consistent with a large number of modes for the rod, and it was not possible to identify the precise modes from the data.

A guided wave can only be excited when the angle of incidence is less than the critical angle, $\theta_{\mathrm{CRIT}}=\arcsin \left(c_{0} / c_{\mathrm{GW}}\right)$. As a result, guided wave artifacts do not necessarily extend to the reflecting tip, as shown in Figure 5. Figure 7A depicts such a case. The guided wave artifact vanishes at an angle of $39.3^{\circ}$, corresponding to a guided wave speed of $2400 \mathrm{~m} / \mathrm{s}$. This is consistent with the slowest measured guided wave speed of 2300 $\mathrm{m} / \mathrm{s}$ (and largest critical angle) shown in Figure 5. The artifact can even be present when the reflecting tip is outside the image (Figure 7B).

Tip Reverberation Artifacts-The tips of cylindrical rods produce a particularly strong reverberation artifact. They appear to be conducive to efficient generation of a specific internal mode, which reverberates to produce a comet tail with periodic banding. This artifact is shown in Figure 8 for a cylindrical rod with a conical tip. We have found that a flat tip produces the same artifact, although the bands are not as strong and distinct. The banding always appears as a short line that is oriented perpendicular to the incident angle of the transducer. The first reflection is almost always very strong (even if the tail is not visible), and it appears that the geometry of the tip acts as an acoustic analogue of a retroreflector.

The periodicity of the banding is directly related to the diameter of the rod, as shown in Figure 8 , in which the diameters of the rods (1.6, 3.2, and $6.4 \mathrm{~mm}$ ) have the same ratio as the periodicity of the banding in the comet tail (1.2, 2.4, and $4.8 \mathrm{~mm}$, respectively). The line spacing of the comet tail corresponds to echo intervals of 1.56, 3.12, and 6.24 microseconds, respectively. The periodicity of the bands implies that just a single mode is responsible for this artifact. For waves that travel straight through the rod, the effective wave speed to produce the measured banding 
would need to be $2.1 \mathrm{~mm} / \mu \mathrm{s}$, much less than the compressional or shear wave speeds in the object, so this signal does not correspond to a simple reverberation model. For waves traveling around the surface of the instrument, the interval corresponds to a circumferential wave speed of $3.2 \mathrm{~mm} / \mu \mathrm{s}$. The speed of a Stonely surface or interface wave ${ }^{58}$ for a stainless steel half-space loaded with water is $2.9 \mathrm{~mm} / \mathrm{s}$. The speed of a circumferential wave ${ }^{20}$ should approach that of the shear wave speed of steel, $3.1 \mathrm{~mm} / \mu \mathrm{s}$. Therefore, the most likely candidate for this artifact is a circumferential wave.

The amplitude of the tip artifact is dependent on the geometry of the tip. Figure 9 shows the tip reverberation artifact at 3 different angles, $\alpha$, between the proximal surface and the acoustic beam. The images show that, for the conical tip, the brightness of the tip artifact is strongest when the proximal surface is at an angle $\alpha \approx 20^{\circ}$ (as shown in Figure 8) and normal to the acoustic beam (Figure 9). It is observable, however, at almost all angles of incidence.

Probe-Instrument Reverberation Artifacts-The reverberation artifacts described above are due to reflection within the instrument. Reverberation can also arise from reflections between the instrument and the probe. This will result in ghost images of the instrument at integer multiples of the true distance between the probe and instrument. Figure 10 shows an image of a cylindrical rod and artifact images at 2 and 3 times the actual depth. Each probe-instrument reverberation artifact produces its own comet tail artifact, and the comet tail artifact is more complex for the ghost images because the incident wave includes the comet tail artifact from the previous surface. These artifacts are only visible when the imaging depth exceeds a multiple of 2 or more times the instrument depth. This artifact may not necessarily obscure features because it will fall in the shadow zone of the instrument. In visualization of $3 \mathrm{D}$ volumes, however, the viewpoint is user selectable; therefore, the location of the shadow zone is not always obvious. In such a case, this artifact could be misconstrued as a real object.

\section{Side Lobe Artifacts}

Diffractive Side Lobe Artifacts-Edges and corners of instruments with radii of curvature on the order of the acoustic wavelength will scatter incident waves over a broad range of angles. Even though the incident energy is redistributed in many directions, the impedance difference between the instrument and tissue is sufficient to produce detectable echoes over all these directions. Figure 11 shows the bright signal from the tip of a conical rod and the curved side lobe artifacts. The artifacts are curvilinear because even when the main lobe is directed away from the tip, the side lobe is incident on the tip and produces an echo. Because the travel time from the tip will

Figure 7. Images of a 3.2-mm-diameter stainless steel rod with a conical tip showing a guided wave reverberation artifact that has separated from the tip. The white lines show the surface of the shaft and the effective "ray" that delineates the edge of the artifact. The angle between the lines was used to determine the critical angle of the guided wave. The arrow denotes the actual location of the tip. A, The tip is in the field of view. B, The tip is out of the field of view, but the guided wave artifact is present in the image.

A

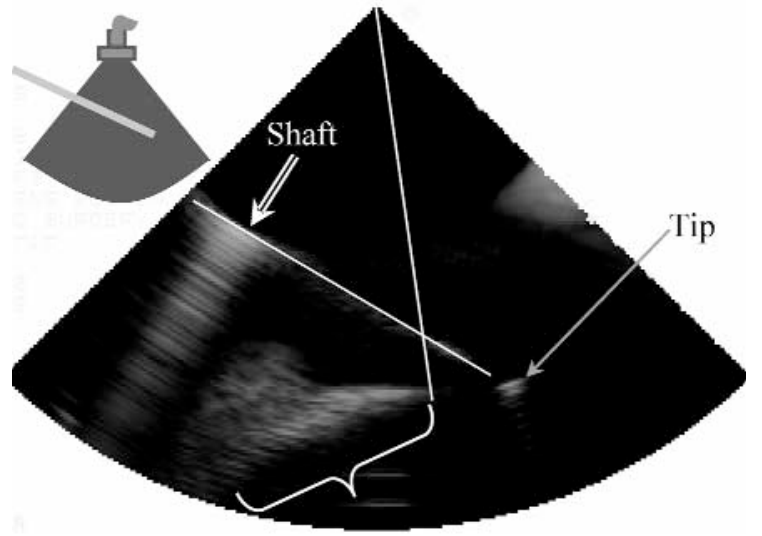

B

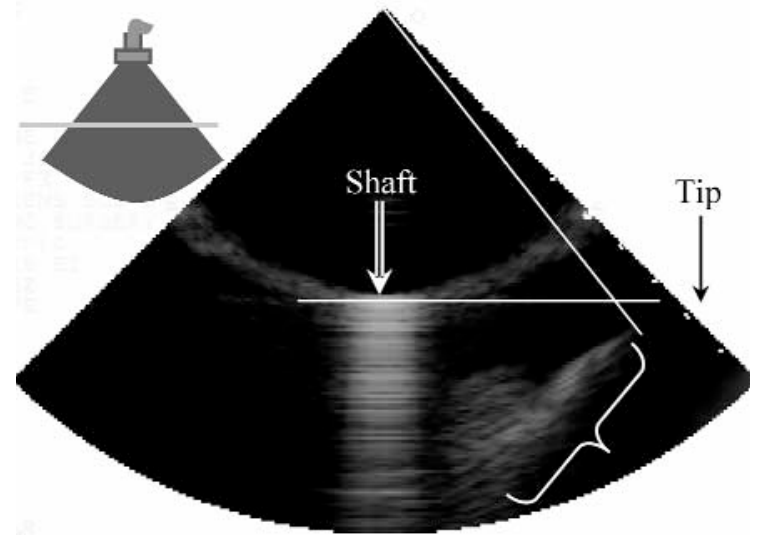


be roughly independent of the direction of the main lobe, the imaging system will place the echo from the tip on an arc of constant radius; hence, in a sector scan, the artifact will be curved, whereas in a linear scan, it should be horizontal.

Figure 8. Effect of rod diameter on tip reverberation artifacts for conically tipped stainless steel rods. A, 1.6-mm-diameter rod. B, 3.2-mm-diameter rod. C, 6.4-mm-diameter rod.

A

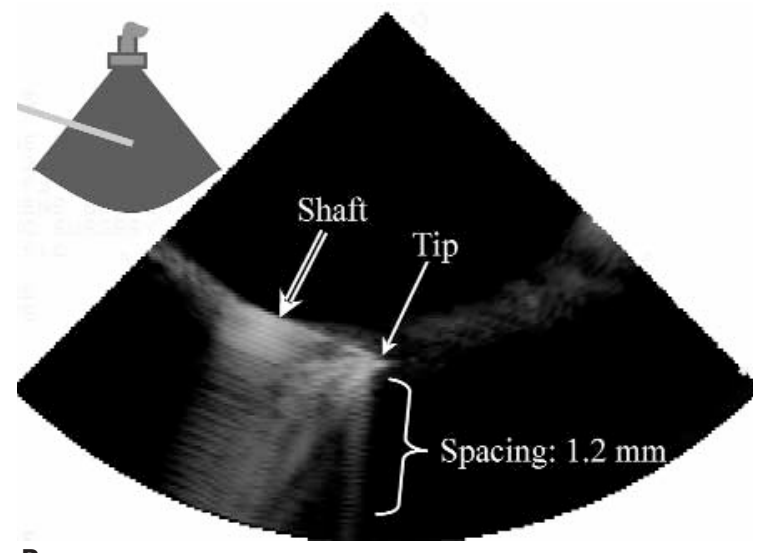

B

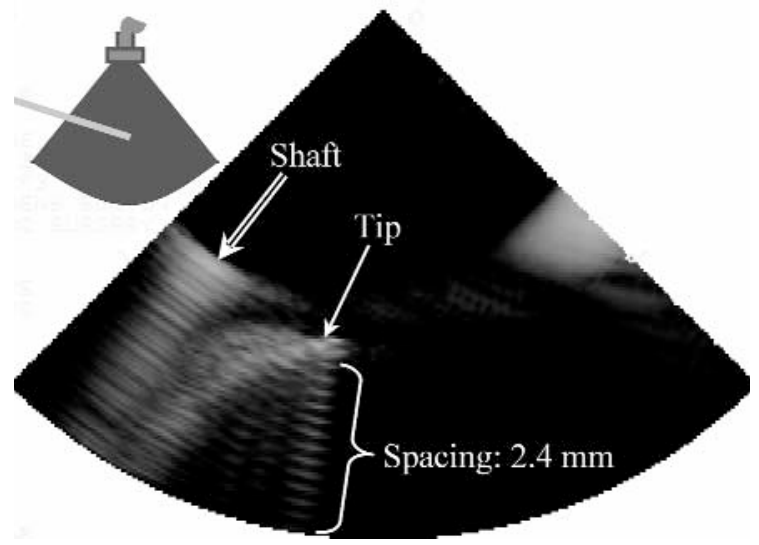

C

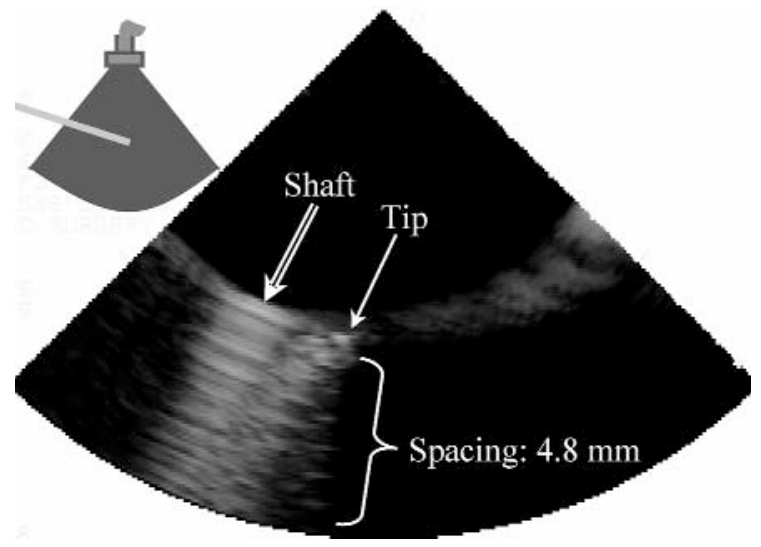

Specular Side Lobe Artifacts-Side lobe artifacts can also arise from other highly reflective instrument surface features. In particular, if a smooth metal surface, which acts as a specular reflector,

Figure 9. Tip reverberation artifact as a function of angle. The angles denoted are the angles between the top proximal surface and the acoustic beam, as shown in the top left corner. $\mathbf{A}, 70^{\circ}$. B, $90^{\circ}$. C, $120^{\circ}$.

A

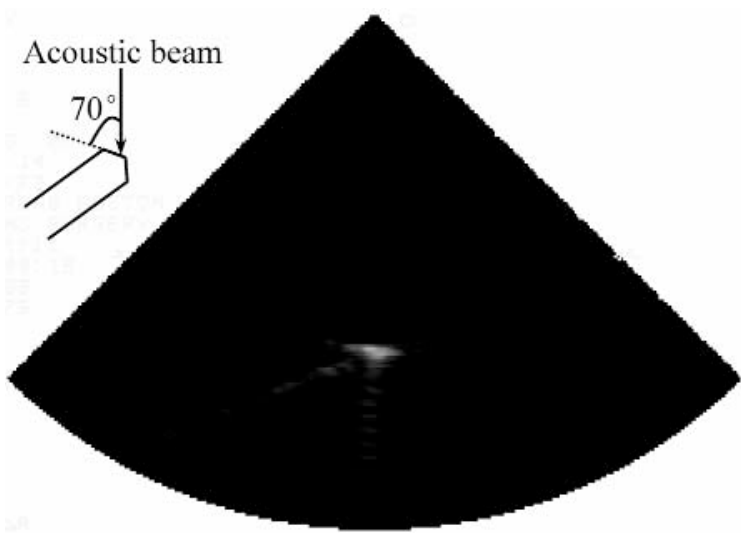

B

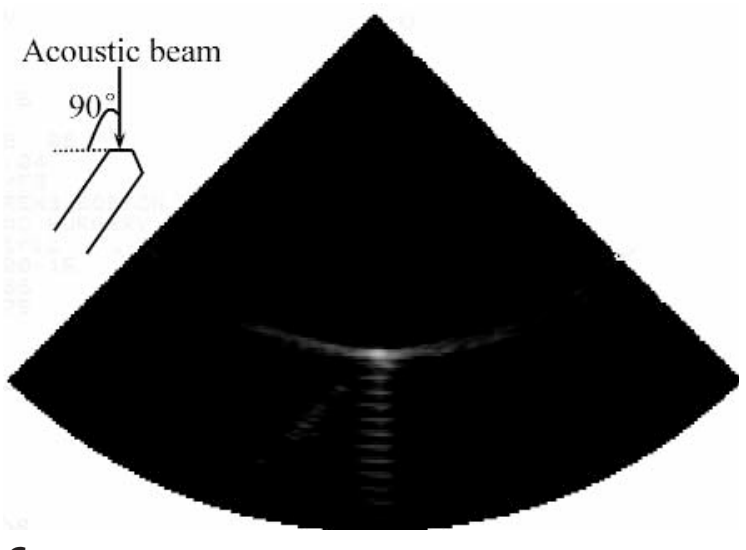

C

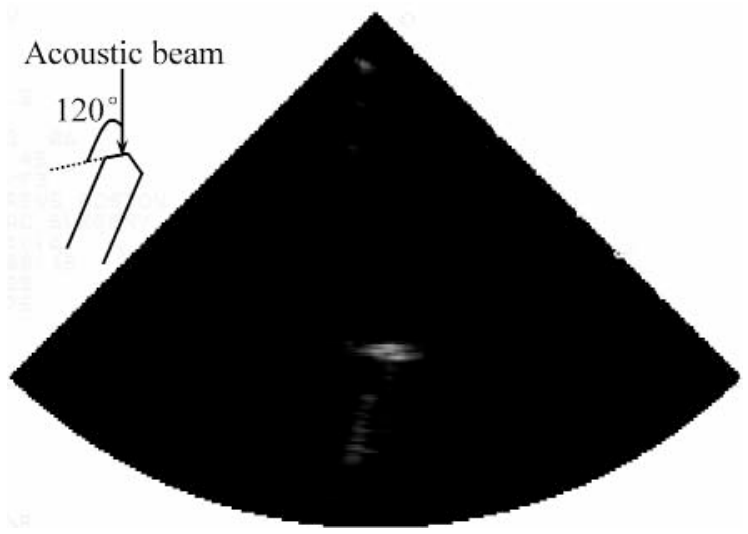




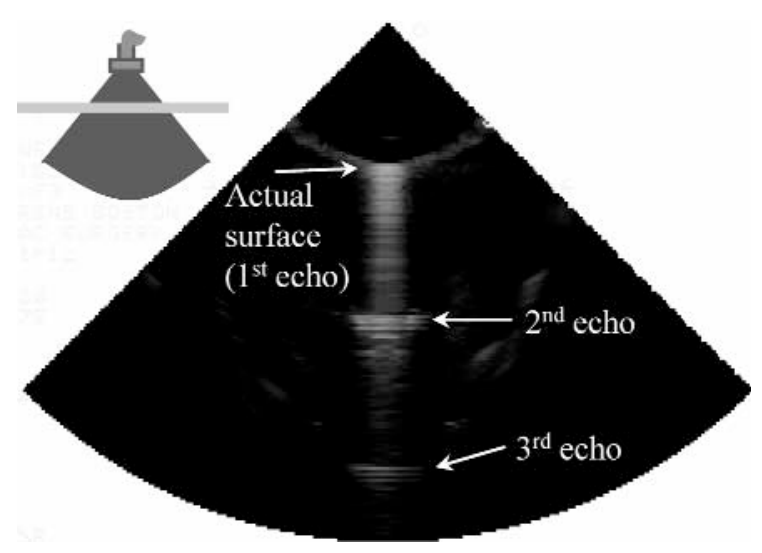

A

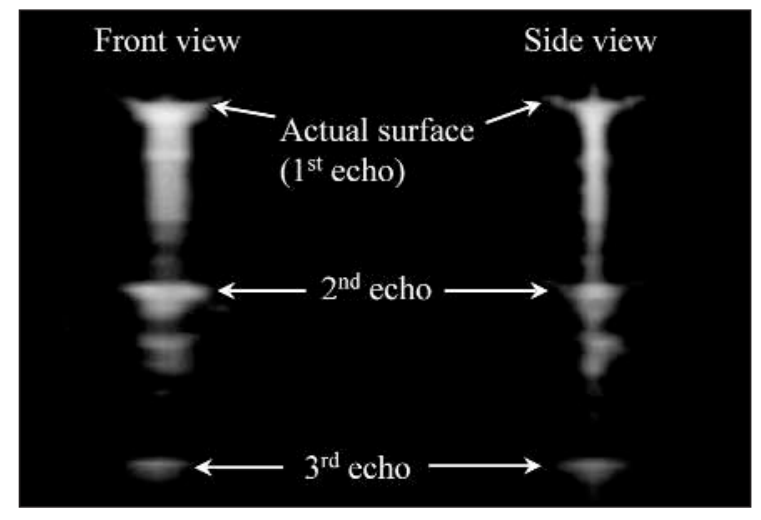

B

Figure 10. Probe-instrument reverberation artifacts for a 3.2-mm-diameter stainless steel rod. A, 2D image. B, 3D images.

is oriented such that a side lobe is normally incident, then it will produce a strong echo signal, which will appear as an artifact. Figure 12 shows this for both a horizontal rod and a rod oriented at $20^{\circ}$.

For the horizontal rod in Figure 12A, the center of the sector scan sees a specular reflection and the comet tail artifact. At the edges of the image, the pulse from the main lobe is reflected away from the probe and does not return an echo. However, a side lobe signal will propagate directly down to the instrument and be reflected back to the scanner. As with the tip artifact, the timing of this echo will be such that it produces a curved artifact on the image. The artifact appears continuous because, as described in the introduction, the short-duration pulses used in imaging systems do not induce the interference patterns observed for a continuous wave source. Note that for this orientation the artifact should not be present for a linear probe because all the lines would be normally incident on the instrument. In Figure 12B, the same effect occurs for a rod at $20^{\circ}$. The specular reflection and comet tail artifact can be seen, and on either side, the curved side lobe artifact is present. In this orientation, a linear scan would also result in an artifact.

\section{Other Types of Artifacts}

Range Ambiguity Artifacts—The range ambiguity artifact occurs when the individual scan lines constituting the B-mode image are generated at a high enough rate that distant echoes from a prior pulse are interpreted as responses to the most recent pulse, and the axial distance from the probe is assigned accordingly. When imaging instruments, this artifact is likely to occur in conjunction with reverberation artifacts. Two likely candidates are the comet tail artifact, which can be very long because of the low inherent absorption of steel, and reverberation between the probe and the instrument. Figure 13 shows an example of an artifactual surface between the probe and the rod. This effect is likely to be most pronounced when the imaging is carried out through a medium with low attenuation, over a short range, and with a high frame rate. It can be reduced or eliminated by decreasing the frame rate.

Figure 11. Diffractive side lobe artifact for a 3.2-mm-diameter stainless steel rod with a conical tip. The artifact appears as curvilinear segments emanating from the tip.

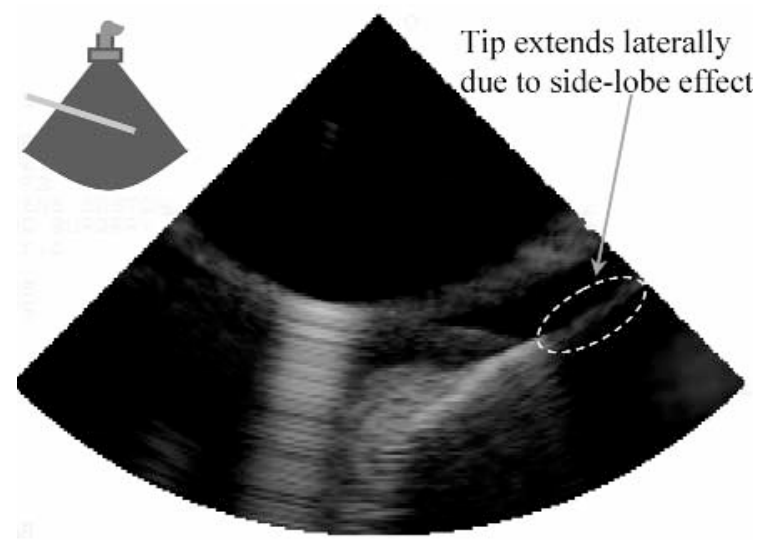




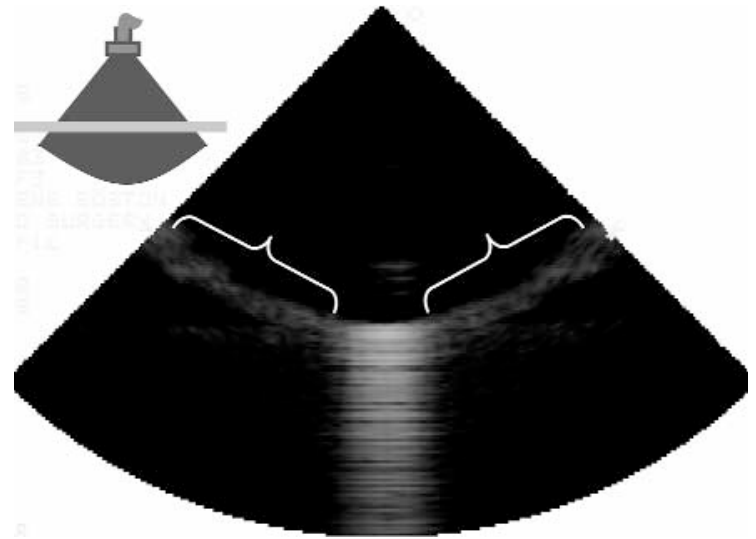

A

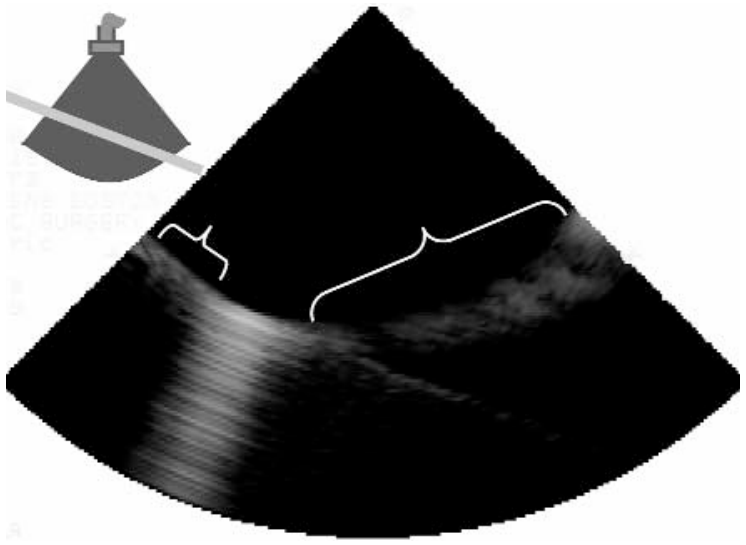

B

Figure 12. Specular side lobe artifacts of a 3.2-mm-diameter stainless steel rod. A, Horizontal rod. B, Rod oriented at $20^{\circ}$.

Mirror Image Artifacts-When instrument surfaces act as specular reflectors, they can enable scatterers that are well outside the acoustic beam to return echoes that will be interpreted as objects within the beam. Figure 14 shows an example with a cylindrical rod placed at $25^{\circ}$ on the left side of the image and a foam scatterer placed just to the right of the center line. A mirror image of the foam can be seen because of pulses that reflect off the rod, scatter off the foam, and reflect off the rod again back to the probe. The time delay of the returning echo is interpreted to produce an image in which the second surface is reflected about the tangent plane of the first surface.

Shadowing-When imaged with tissue or other objects, metal instruments, like bone, create shadow regions in ultrasound images. These arise because those sound waves that are transmitted through the front and back surfaces of an instrument are not of sufficient intensity to reflect off tissue and pass back through the instrument to reach the probe. Figure 15 shows a pronounced shadow created by a stainless steel rod on a planar cloth surface.

\section{Artifacts of Minimally Invasive Instruments}

The rods used to illustrate the artifacts described above are typical of the shafts of most minimally invasive instruments. Although the tools located at the distal ends of these instruments often possess greater geometric complexity than the shafts, the artifacts they produce are similar. In this section, images of 3 instruments are presented, and it is shown that their artifacts can be categorized using the artifacts observed for rods. Figure 16 shows both 2D and 3D water tank images of the 19-gauge needle, suturing device, and forceps displayed in Figure 3. The artifacts observed with rods can be seen in these images. The images of the 19-gauge needle (Figure 16A and Video 1) include a comet tail artifact, 3 guided wave artifacts, a tip reverberation artifact, a diffractive side lobe artifact, and a specular side lobe artifact.

The suturing device (Figure 16B and Video 2) produced comet tail, guided wave, specular side lobe, diffractive side lobe, and tip reverberation artifacts. For this device, the reverberation artifacts are not as distinct as those of the needle.

Figure 13. Range ambiguity artifact for a 3.2-mm-diameter stainless steel rod.

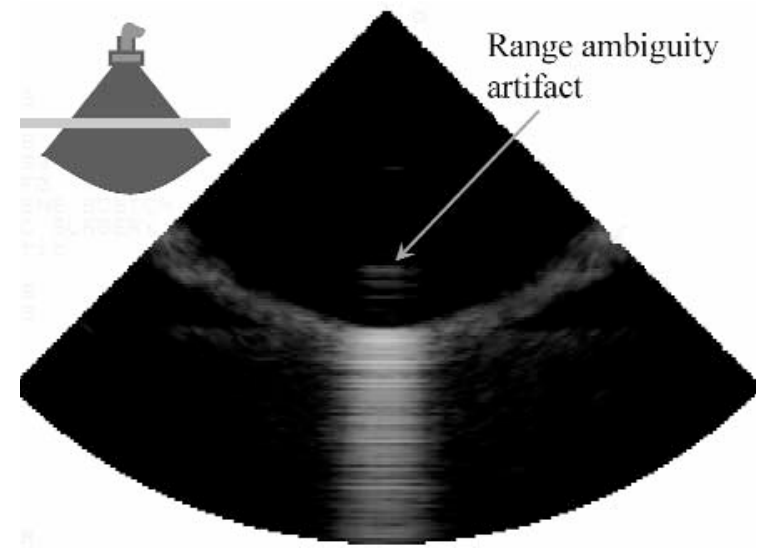


This is likely due to the main shaft's being constructed from 2 semicylinders that introduce an extra boundary for reflections. In addition, bubbles can be trapped between the 2 semicylinders and produce a ring-down artifact. In the vicinity of the slot (where the needle is), the artifacts make visualizing the needle and slot almost impossible.

The forceps (Figure 16C and Video 3) also produced comet tail, guided wave, and specular side lobe artifacts, although, as with the suturing device, they were not as clear as the needle. The forceps produces a reverberation band behind the joint (where the fingers connect to the shaft), which may be due to a comet tail artifact or could be due to ring-down associated with air bubbles in the joint. The 2 fingers of the forceps are tapered semicylinders with ridged grasping surfaces. The ridges form a rough surface, which may reduce the patterned reverberation bands in the finger while diffracting sound to the neighboring edges, which may produce echoes back to the transducer. The result is a cloud underneath the front smooth surface of the finger (Figure 16C, finger 1), and the tip of the finger does not produce a strong artifact.

These images are of isolated instruments in a water tank, which is a near ideal imaging environment. In vivo conditions are much less ideal, as shown by the images in Figure 17 and Videos 4-7, depicting these tools in the right atrium of a beating pig heart. As shown, the combination of reflections from an instrument and tissue

Figure 14. Mirror-image artifact produced by a 3.2-mm-diameter stainless steel rod with a piece of polyurethane foam.

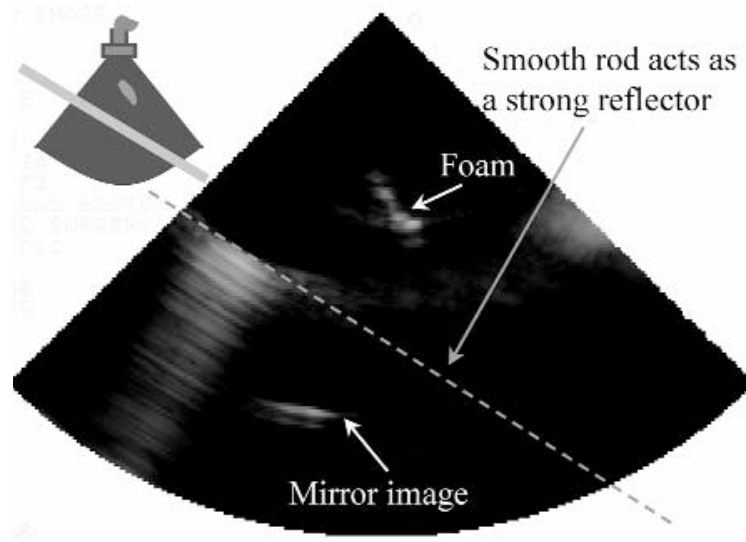

produces images that are very hard to decipher. For example, the guided wave artifacts from the tip of the needle (Figure 17B) resemble the for-

Figure 15. Shadowing from a stainless steel rod on a cloth surface. A, 2D image of the cloth alone. B, 2D image of a stainless steel rod on top of the cloth. C, 3D image of a stainless steel rod on top of the cloth.

\section{A}

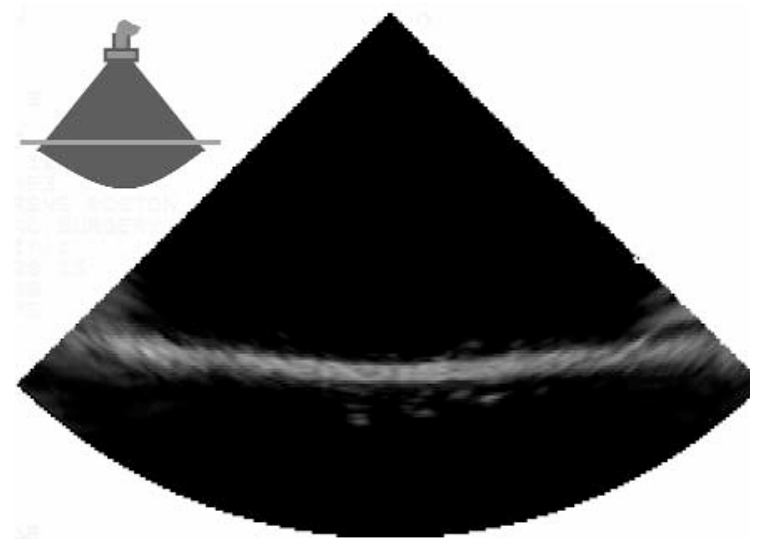

B

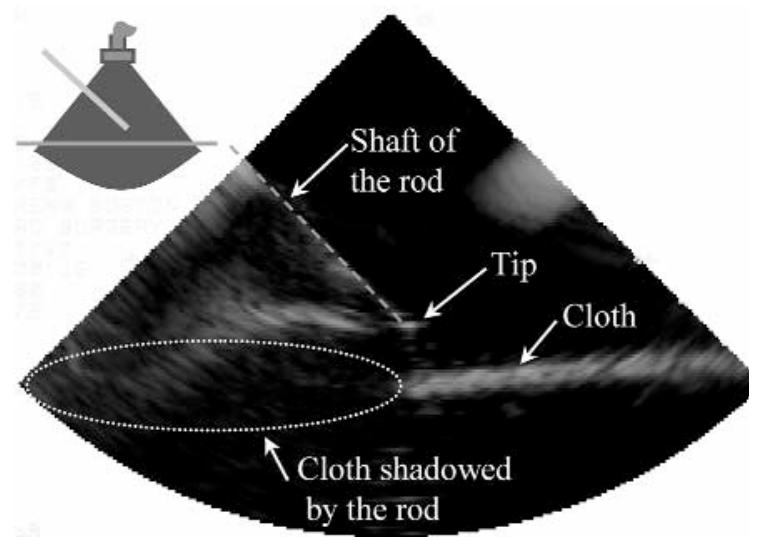

C

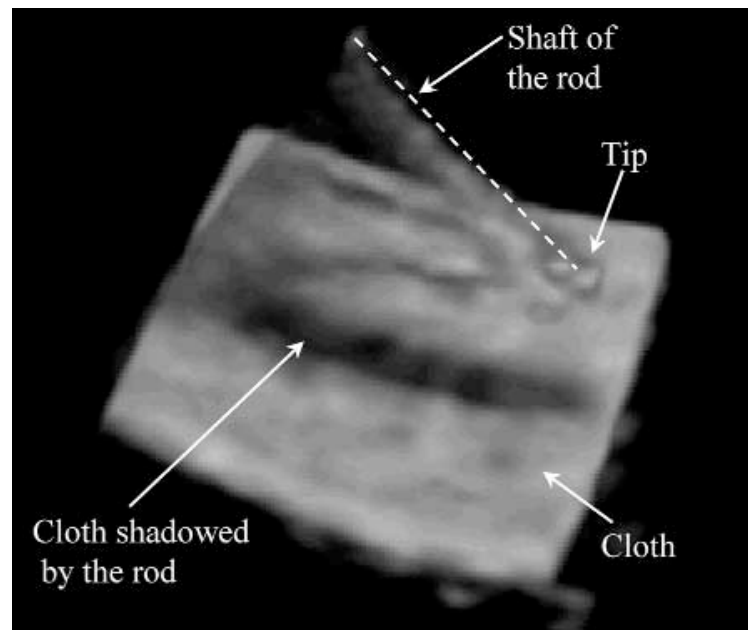


ceps' fingers. Reverberation artifacts from the suturing device (Figure 17C) completely obscure the tissue. The forceps itself also obscures the tissue and produces ghost images due to reverberation between the instrument and the probe.

\section{Discussion}

Image-based interventions involving the cutting, removal, or approximation of tissue require precise interactions between the instruments and tissue. Instrument-tissue positioning require-

Figure 16. Clinical instrument images in water. A, 19-gauge needle. B, Suturing device with a needle. C, Forceps. Labeled artifacts: CT, comet tail; DSL, diffractive side lobe; GW, guided wave; SSL, specular side lobe; and TR, tip reverberation.

A

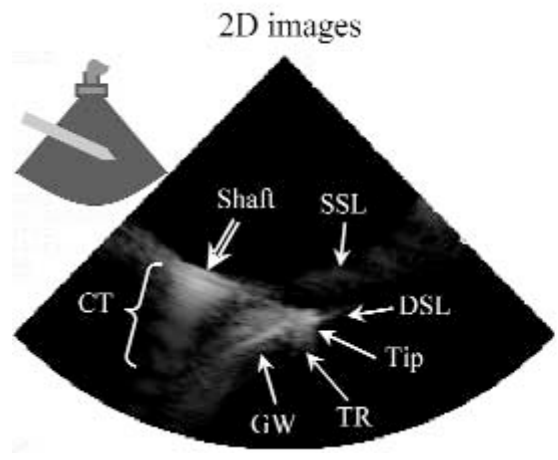

3D images
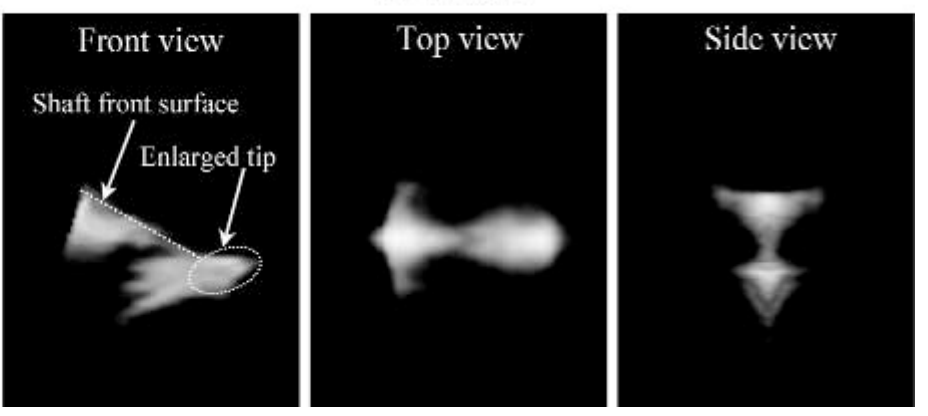

B
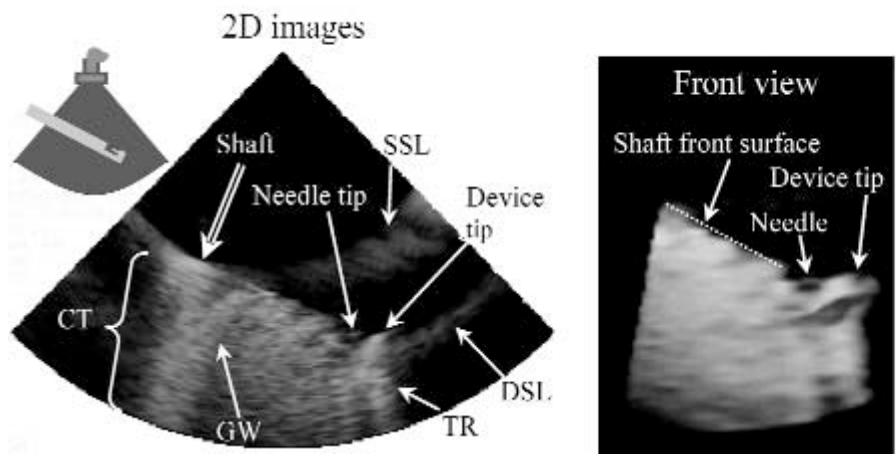

3D images
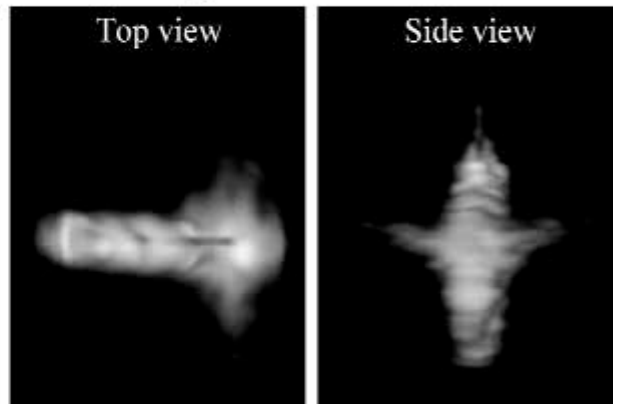

C
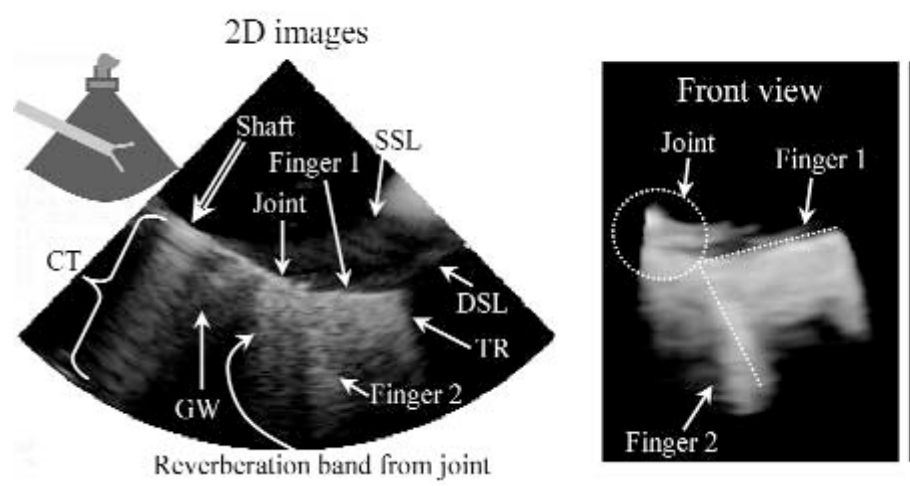

3D images
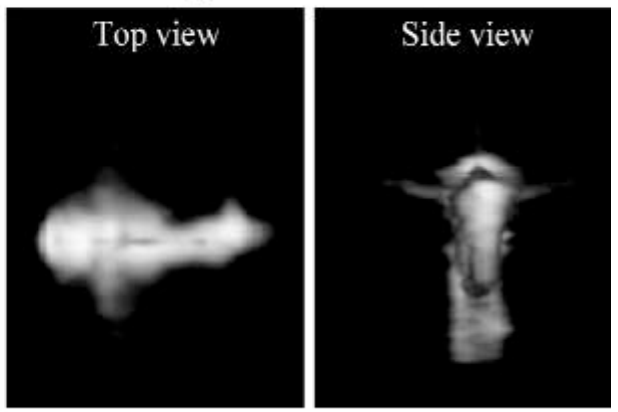
ments are often on the order of $1 \mathrm{~mm}$, and the clinician must be able to recognize when instrument-tissue contact is made. In some procedures, contact forces must be controlled such that contact is maintained or desired tissue deformation is achieved without tissue damage and despite tissue motion due to physiologic forces. Given the limited tactile feedback available during minimally invasive procedures, imaging is heavily relied on to guide these instrument-tissue interactions.

As shown above, metallic instruments imaged under ultrasound generate a large number of very strong artifacts that make visualization of instruments, even in ideal conditions, very challenging. A number of these artifacts have been observed in diagnostic imaging - and indeed are often used in the diagnostic evaluation-but in instruments, the artifacts are more pronounced and often fill a larger fraction of image space. A variety of techniques can be used to mitigate these artifacts. These include (1) probe placement and viewpoint selection, (2) use of artifacts to infer instrument location, and (3) instrument modification. Each is discussed briefly below.

\section{Probe Placement and Viewpoint Selection}

Given a set of instruments and the tissue manipulations necessary for a procedure, one can select the relative locations of the instruments, tissue, and probe to minimize the intensity of artifacts or to position them outside the image

Figure 17. Three-dimensional images of instruments in the right atrium of a beating porcine heart. A, Tissue image without an instrument inserted. B, 19-gauge needle. C, Suturing device. D, Forceps. Artifact identifiers: CT, comet tail; GW, guided wave; SSL, specular side lobe; and TR, tip reverberation.

A

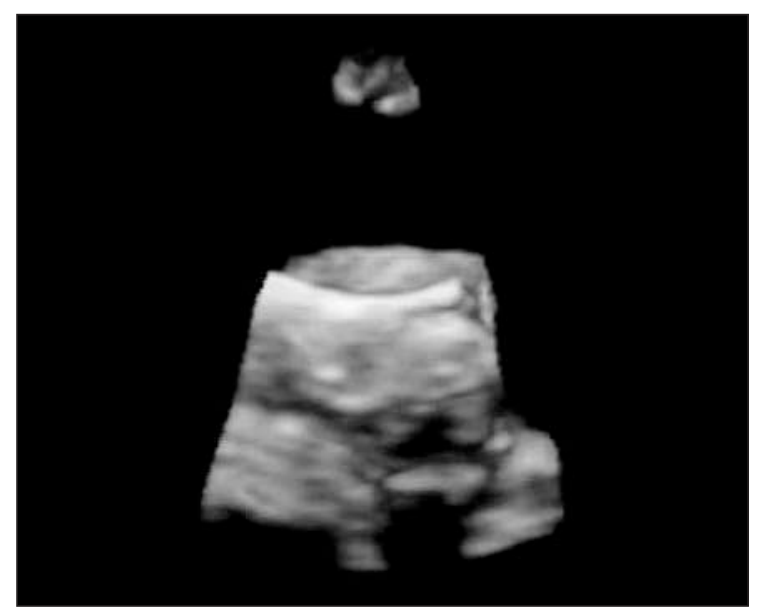

C

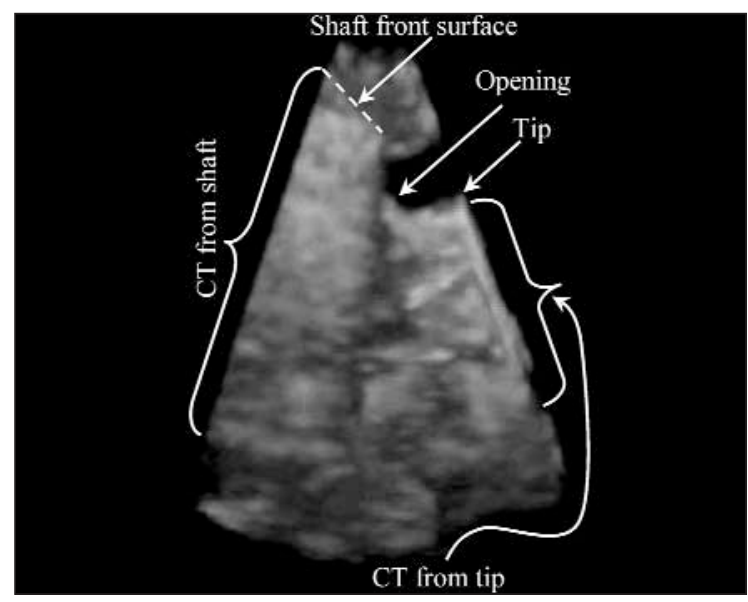

B

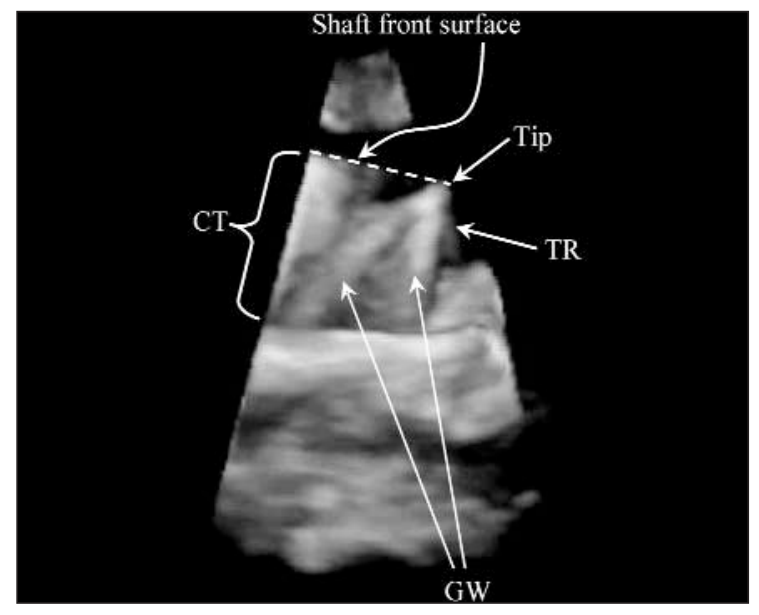

D

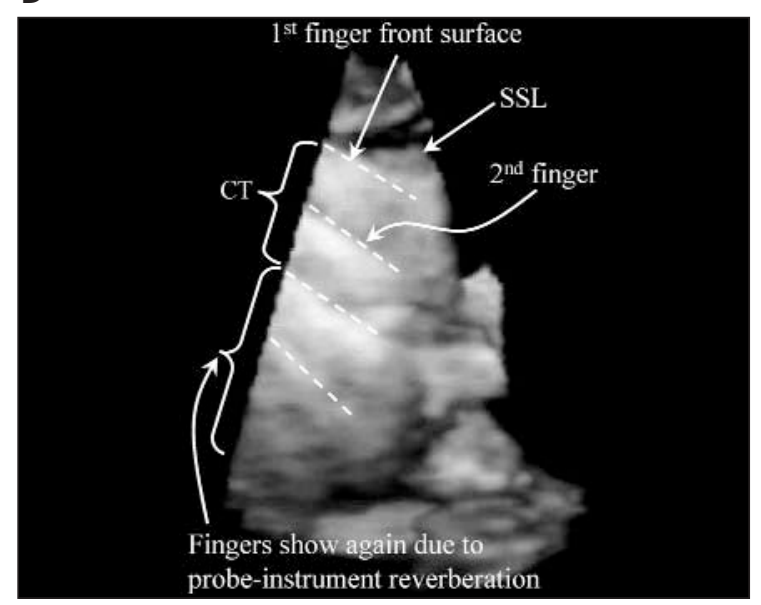


region of tool-tissue interaction. For example, angling the instrument so that it is not perpendicular to the scan lines ensures that comet tail artifacts do not occur in this region. Optimizing the relative positions of the instruments and probe is, however, often highly constrained by the anatomy; for example, instruments cannot be inserted through organs, delicate tissue, and bone. Nonetheless, considerable improvements in image quality are often possible with this approach.

One such technique that can be used even when the probe and instruments must be positioned close to each other is to place artifacts in the shadow of an instrument. In the absence of artifacts, these shadows appear as dark silhouettes on tissue located on the far side of an instrument from the probe. All the reverberation artifacts described in the article as well as mirror image artifacts lie in the shadow region of the instrument. Given their location, they cannot represent any real object, instrument or tissue, and so can be ignored.

In 2D ultrasound images, the operator can visually determine where the shadow region lies and deliberately ignore its image contents. A more complicated approach using instrument tracking and image processing would be to darken all image pixels in the shadow region. An alternate approach is possible with 3D imaging systems. These systems allow the user to select an arbitrary viewpoint orientation with respect to the probe. By selecting the viewpoint to correspond to that of the probe, instrument shadows and any artifacts they contain lie behind the instruments and so are hidden from view as part of the image rendering. For example, Figure 18 presents the image of Figure 1 when viewed from the probe.

\section{Inferring Instrument Location From Artifacts}

In many cases, the artifacts in an image can be more visible than the instruments themselves. In these situations, the location and motion of the instruments can often be inferred from knowledge of how artifacts evolve as the relative angles between an instrument and the probe vary. Comet tail artifacts, for example, reveal the normal surface of the instrument shaft. Guided wave artifacts point to the location of an instru- ment's tip, and the tip scattering that produces diffractive side lobe artifacts also ensures that the tip is highly visible. Deliberate motion of an instrument with respect to the surrounding tissue is often helpful in determining instrument location and also for distinguishing instrument artifacts from tissue structures. The value of this technique is substantial and cannot be adequately conveyed in a printed document.

The relative location of an instrument and its artifacts can also be used to infer location. For example, when an instrument is inserted in a fluid-filled lumen, such as the heart, the distance between the instrument's image and its shadow on the lumen wall corresponds to the actual distance between them. Tissue contact occurs when the instrument touches its shadow.

\section{Artifact Reduction Through Instrument Modification}

Modification techniques that reduce the intensity and specularity of instrument echoes can improve the image produced by the initial echo from the central beam lobe as well as reduce or eliminate artifacts arising from multiple reflections and side lobe echoes. The most drastic approach to modification is to redesign instruments using materials other than metal. Simpler modifications to existing instruments involve changing the surface finish or adding a coating to the surface. Several examples of alternate materials are presented in Figure 19. Materials with an acoustic impedance closer to that of tissue will produce fewer and less intense artifacts than metals such as stainless steel. The copolymer and fiberglass rods, for example, show comet tail and tip artifacts similar to those of steel, but the extent of the artifact is dramatically less than that of steel. If a material is also a diffusive reflector, such as the Franklin fiber (Figure 19D), it will be highly visible as well.

Modifying the surfaces of existing metal instruments, either through surface finish or by adding a coating, can also lead to a substantial reduction of artifacts, as shown in Figure 20 and Videos $8-11$. A roughened surface (Figure 20B) was able to reduce reverberation artifacts of a stainless steel instrument to those seen with the copolymer or fiberglass rods. The rough surface distorts the wave front entering and leaving the instru- 


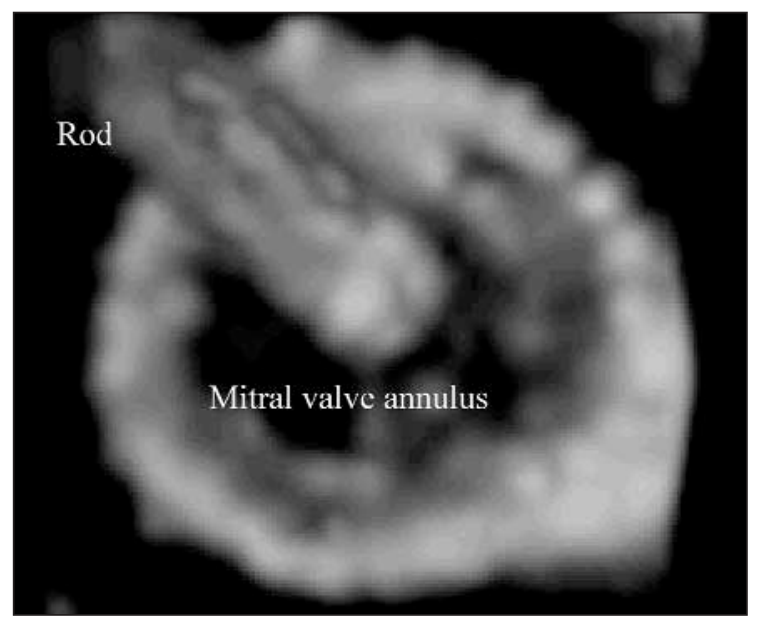

Figure 18. Image of a rod positioned in the center of a porcine in vitro pulsatile mitral valve model, as described in Figure 1, where the image-rendering viewpoint was selected to place artifacts in the instrument shadow. ment and so prevents multiple reflections from producing coherent echoes at the probe. The roughness may also reduce the sharpness of an edge and make it a weaker scatterer and thus may reduce the side lobe effect from the edge. In addition, diffusive scattering from a rough surface will enhance surface visibility at non-normal angles of insonification. It is also likely, however, to produce some side lobe artifacts because a rough metal surface is still a much stronger scatterer than tissue.

An absorptive coating can be added to a rough surface to further reduce the side lobe artifacts and residual reverberation (Figure 20, D and F). Alternatively, a coating that is both diffusive and absorptive will both enhance visibility and reduce artifacts; for example, polyurethane foam on a smooth steel surface (Figure 20E) results in

Figure 19. Effect of material on imaging for a 3.2-mm-diameter rod in water with its axis at $15^{\circ}$ from horizontal. A, Stainless steel. B, Acetyl copolymer. C, Fiberglass. D, Vulcanized Franklin fiber processed from cotton.

A

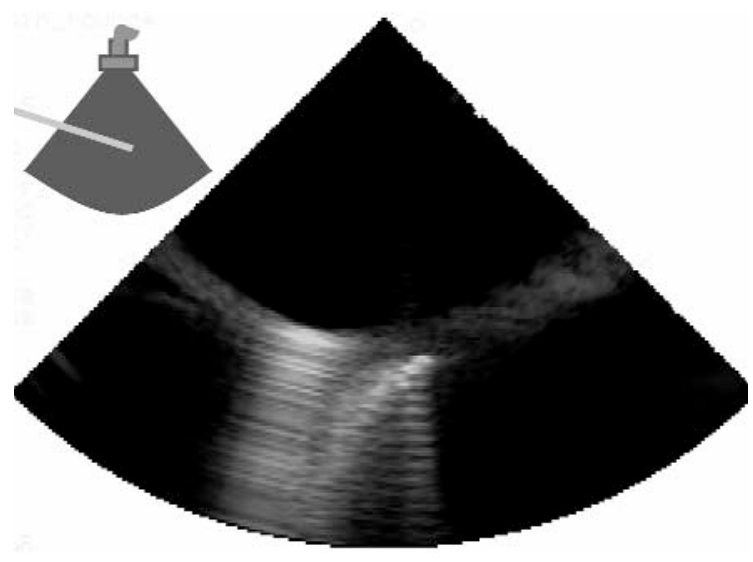

C

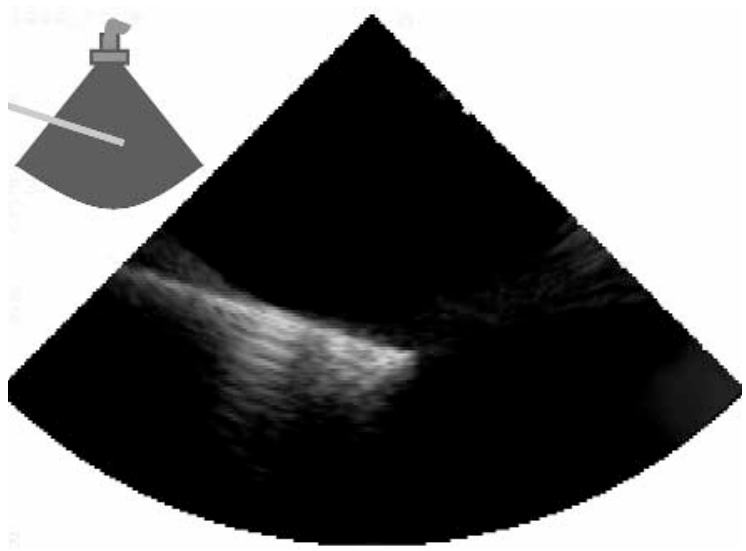

B

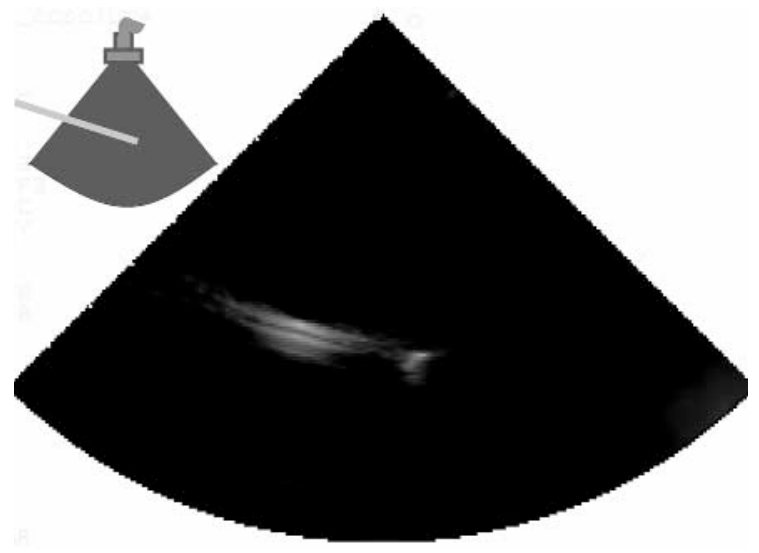

D

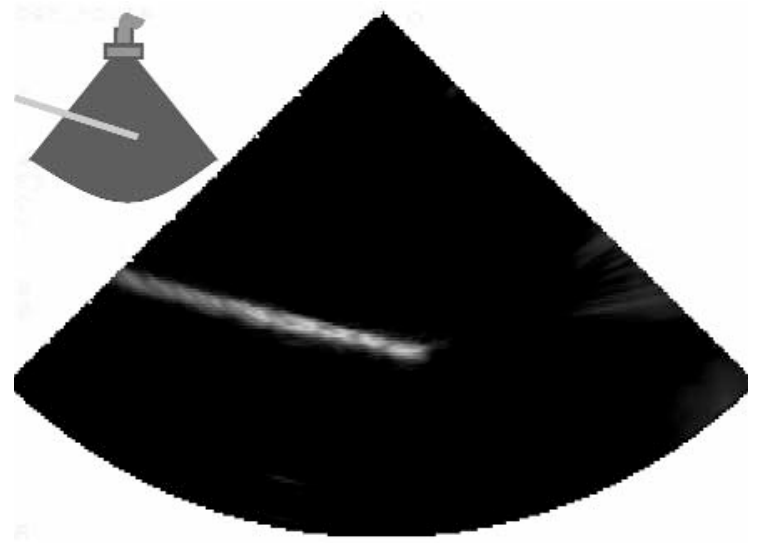


an excellent image of the rod. An additional advantage of this approach is that coating the surface of an existing instrument is typically simpler and less expensive than mechanically roughening its surface. As an example, images of a suturing device coated with polyurethane foam are shown in Figure 21 and Video 12. The images of the coated device result in a faithful reproduction of the instrument, and the large reverberation artifacts of Figure 16B are absent. The notch

Figure 20. Effect of surface treatment on imaging for a 3.2-mm-diameter stainless steel rod in water with its axis at $15^{\circ}$ from horizontal. A, Smooth surface. B, Threaded surface. C, Smooth surface coated with Teflon $(0.03 \mathrm{~mm}$ thick). D, Threaded surface coated with Teflon $(0.09 \mathrm{~mm}$ thick). E, Smooth surface coated with polyurethane foam ( $0.8 \mathrm{~mm}$ thick). F, Threaded surface coated with polyvinylidene difluoride $(0.14 \mathrm{~mm}$ thick).

A

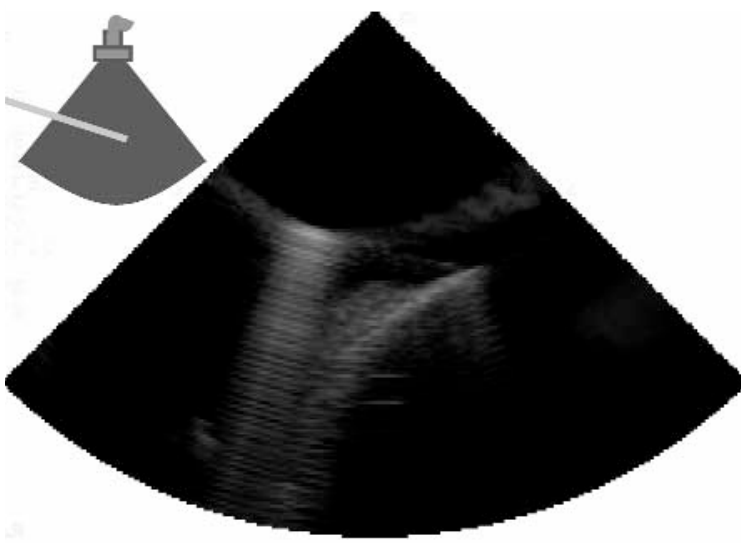

C

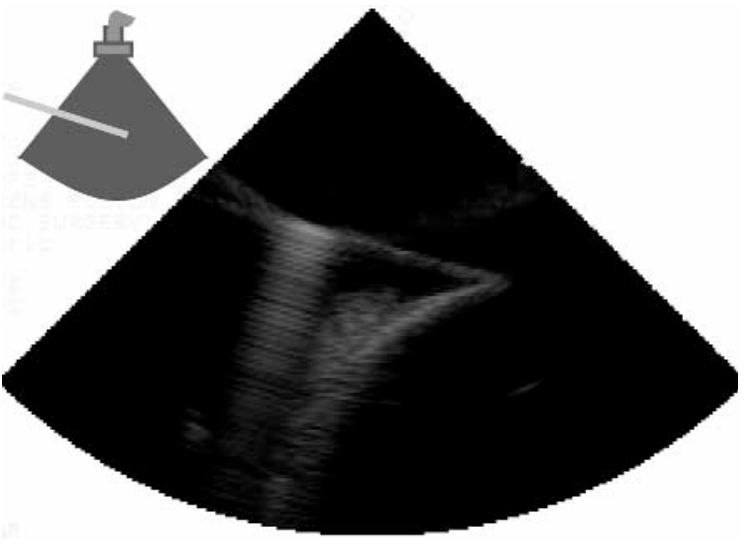

E

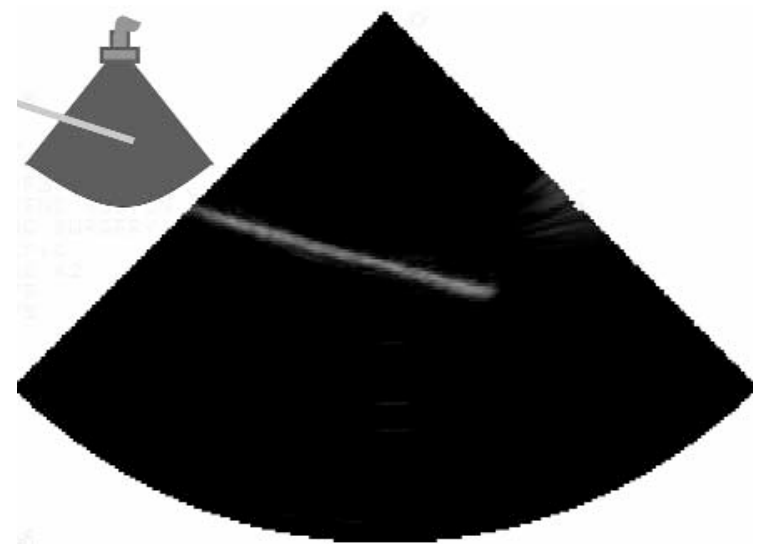

B

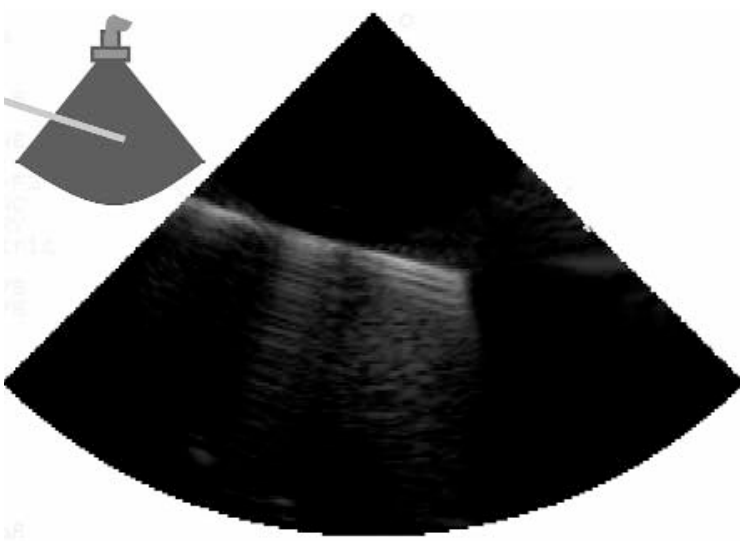

D

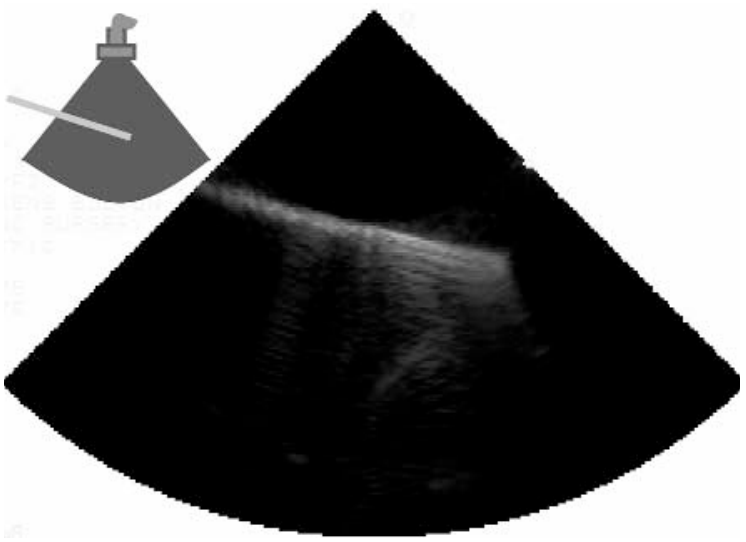

$\mathbf{F}$

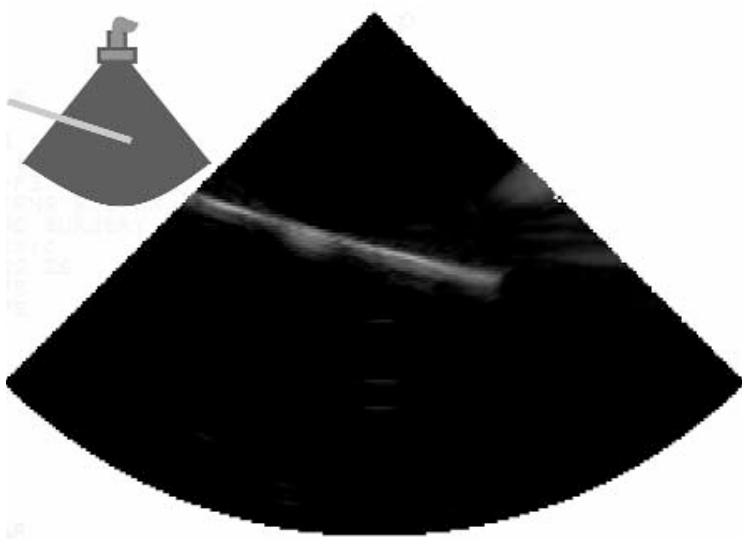



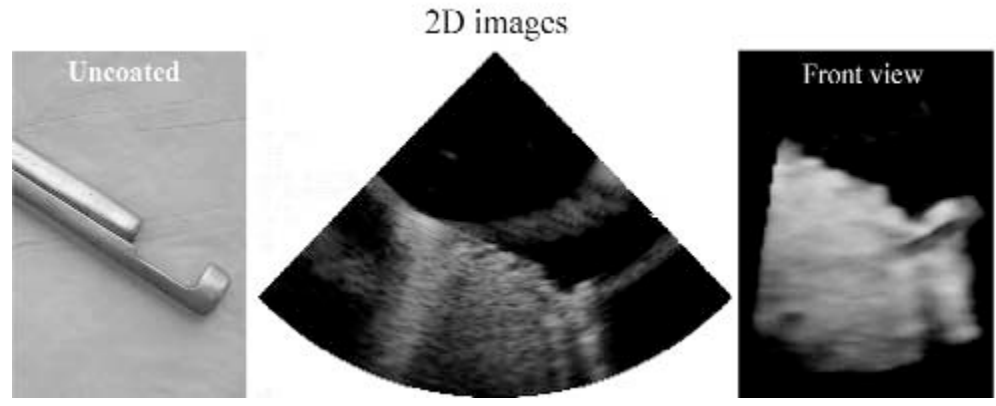

3D images

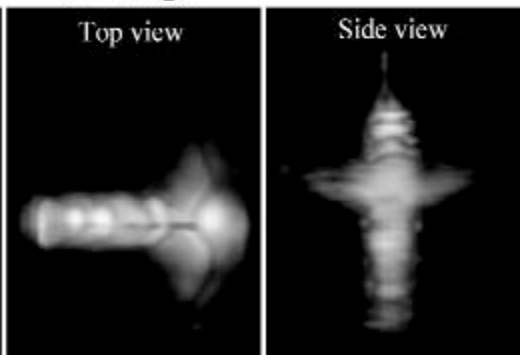

A
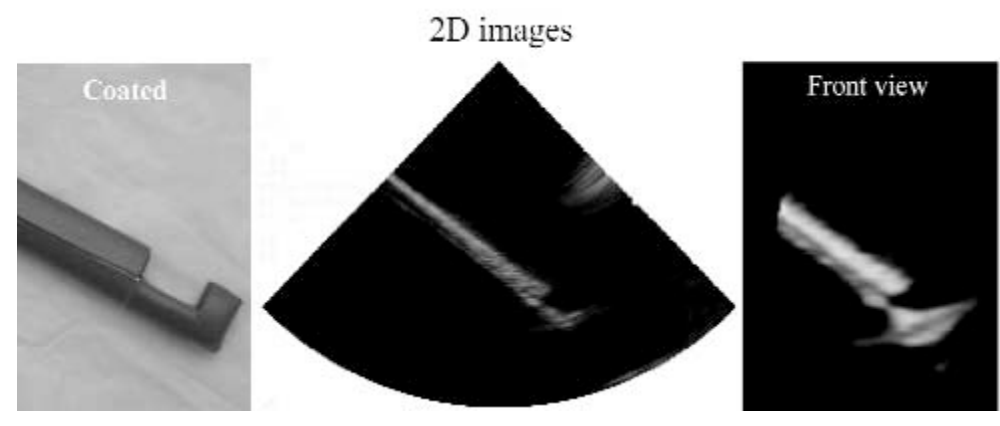

3I) images
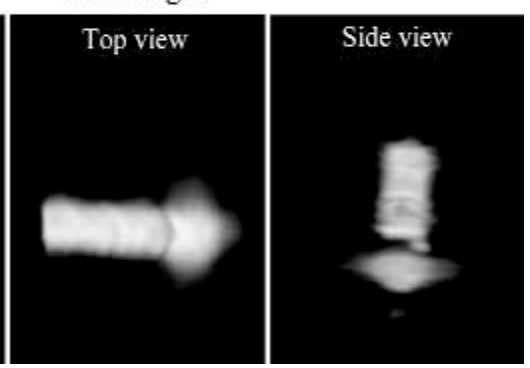

Figure 21. Effect of polyurethane foam coating on suturing device imaging. A, Uncoated. B, Coated with polyurethane foam.

is clearly visible, and the curvilinear artifacts that emanated from the tip of the untreated instrument are dramatically reduced.

Given the benefits of ultrasound imaging and the increasing availability and versatility of 3D ultrasound systems, it is likely that the number and complexity of ultrasound-guided interventions will continue to increase in the years ahead. Although imaging of instrument-tissue interactions will be a substantial challenge in the development of these procedures, this article provides a framework for addressing this challenge. Categorization of instrument artifacts according to their underlying acoustic phenomena provides a mechanism for predicting under what conditions they will interfere with a procedure. Furthermore, this understanding can be used to guide the procedure and instrument design processes.

\section{References}

1. Matalon TA, Silver B. US guidance of interventional procedures. Radiology 1990; 174:43-47.

2. Dodd GD III, Esola CC, Memel DS, et al. Sonography: the undiscovered jewel of interventional radiology. Radiographics 1996; 16:1271-1288.
3. Holm HH, Skjoldbye B. Interventional ultrasound Ultrasound Med Biol 1996; 22:773-789.

4. Campani R, Bottinelli O, Calliada F, Coscia D. The latest in ultrasound: three-dimensional imaging, part II. Eur J Radiol 1998; 27(suppl 2):S183-S187.

5. Downey DB, Fenster A, Williams JC. Clinical utility of threedimensional US. Radiographics 2000; 20:559-571.

6. Cannon JW, Stoll JA, Salgo IS, et al. Real-time three-dimensional ultrasound for guiding surgical tasks. Comput Aided Surg 2003; 8:82-90.

7. Suematsu Y, Marx G, Stoll J, et al. Three-dimensional echocardiography-guided beating heart surgery without cardiopulmonary bypass: a feasibility study. J Thorac Cardiovasc Surg 2004; 128:579-587.

8. Suematsu Y, Martinez JF, Wolf BK, et al. Three-dimensional echo-guided beating heart surgery without cardiopulmonary bypass: atrial septal defect closure in a swine model. J Thorac Cardiovasc Surg 2005; 130:1348-1357.

9. Vasilyev NV, Martinez JF, Freudenthal FP, Suematsu Y, Marx GR, del Nido PJ. Three-dimensional echo and videocardioscopy-guided atrial septal defect closure. Ann Thorac Surg 2006; 82:1322-1326.

10. Kremkau FW, Taylor KJW. Artifacts in ultrasound imaging. J Ultrasound Med 1986; 5:227-237.

11. Evans RG. Medical diagnostic ultrasound instrumentation and clinical interpretation: report of the ultrasonography task force. Council on Scientific Affairs. JAMA 1991; 265:1155-1159. 
12. Scanlan KA. Sonographic artifacts and their origins. AJR Am J Roentgenol 1991; 156:1267-1272.

13. Kossoff G. Basic physics and imaging characteristics of ultrasound. World J Surg 2000; 24:134-142.

14. Nelson TR, Pretorius DH, Hull A, Riccabona M, Sklansky MS, James G. Sources and impact of artifacts on clinical three-dimensional ultrasound imaging. Ultrasound Obstet Gynecol 2000; 16:374-383.

15. Nilsson A. Artefacts in sonography and Doppler. Eur Radiol 2001; 11:1308-1315.

16. Faran JJ Jr. Sound scattering by solid cylinders and spheres. J Acoust Soc Am 1951; 23:405-418.

17. Neubauer WG, Dragonette LR. Observation of waves radiated from circular cylinders caused by an incident pulse. J Acoust Soc Am 1970; 48:1135-1149.

18. Schuetz LS, Neubauer WG. Acoustic reflection from cylinders: nonabsorbing and absorbing. J Acoust Soc Am 1977; 62:513-517.

19. Hefner LV, Goldstein A. Resonance by rod-shaped reflectors in ultrasound test objects. Radiology 1981; 139:189-193.

20. Fan $Y$, Honarvar F, Sinclair AN, Jafari MR. Circumferential resonance modes of solid elastic cylinders excited by obliquely incident acoustic waves. J Acoust Soc Am 2003; 113:102-113.

21. Wendell BA, Athey PA. Ultrasonic appearance of metallic foreign bodies in parenchymal organs. J Clin Ultrasound 1981; 9:133-135.

22. Ziskin MC, Thickman DI, Goldenberg NJ, Lapayowker MS, Becker JM. The comet tail artifact. J Ultrasound Med 1982; 1:1-7.

23. Thickman DI, Ziskin MC, Goldenberg NJ, Linder BE. Clinical manifestation of the comet tail artifact. J Ultrasound Med $1983 ; 2: 225-230$

24. De Flaviis L, Scaglione P, Del Bò P, Nessi R. Detection of foreign bodies in soft tissues: experimental comparison of ultrasonography and xeroradiography. J Trauma 1988; 28 : 400-404.

25. Shapiro RS, Winsberg F. Comet-tail artifact from cholesterol crystals: observations in the postlithotripsy gallbladder and an in vitro model. Radiology 1990; 177:153-156.

26. Lichtenstein D, Mézière G, Biderman P, Gepner A, Barré O. The comet-tail artifact. Am J Respir Crit Care Med 1997; 156:1640-1646.

27. Avruch L, Cooperberg PL. The ring-down artifact. J Ultrasound Med 1985; 4:21-28.

28. Lim JH, Lee KS, Kim TS, Chung MP. Ring-down artifacts posterior to the right hemidiaphragm on abdominal sonography: sign of pulmonary parenchymal abnormalities. J Ultrasound Med 1999; 18:403-410.

29. Kohzaki S, Tsurusaki K, Uetani M, Nakanishi K, Hayashi K. The aurora sign: an ultrasonographic sign suggesting parenchymal lung disease. Br J Radiol 2003; 76:437-443.
30. Meeker TR, Meitzler AH. Guided wave propagation in elongated cylinders and plates. In: Mason WP (ed). Physical Acoustics. New York, NY: Academic Press; 1964.

31. Zemanek J Jr. An experimental and theoretical investigation of elastic wave propagation in a cylinder. J Acoust Soc Am 1971; 51:265-283.

32. Szabo T. Array beamforming. In: Diagnostic Ultrasound Imaging: Inside Out. Burlington, MA: Elsevier; 2004:171212.

33. Blackstock DT. Arrays. In: Fundamentals of Physical Acoustics. New York, NY: Wiley-Interscience; 2000:495509.

34. Laing F, Kurtz AB. The importance of ultrasonic side-lobe artifacts. Radiology 1982; 145:763-768.

35. Abbott JG, Thurston FL. Acoustic speckle: theory and experimental analysis. Ultrason Imaging 1979; 1:303-324.

36. Wells PNT, Halliwell DJ. Speckle in ultrasound imaging. Ultrasonics 1981; 19:225-229.

37. Thijssen JM, Oosterveld BJ. Speckle and texture in echography: artifact or information? IEEE Ultrasonics Symp Proc 1986; 803-809.

38. Gardner FJ, Clark RN, Kozlowski R. A model of a hepatic mirror-image artifact. Med Ultrasound 1980; 4:19-21.

39. Wittich G, Czembirek H, Tscholakoff D. Retrocaval pseudolymphoma: Clinical impact of the mirror artifact. J Ultrasound Med 1982; 1:173-176.

40. Cakmakci H, Gulcu A, Zenger MN. Mirror-image artifact mimicking epidural hematoma: usefulness of power Doppler sonography. J Clin Ultrasound 2003; 31:437-439.

41. Sommer FG, Filly RA, Minton MJ. Acoustic shadowing due to refractive and reflective effects. AJR Am J Roentgenol 1979; 132:973-977.

42. Robinson DE, Wilson LS, Kossoff G. Shadowing and enhancement in ultrasonic echograms by reflection and refraction. J Clin Ultrasound 1981; 9:181-188.

43. Ziskin MC, LaFollette PS, Radecki PD, Villafana T. The retrolenticular afterglow: an echo enhancement artifact. J Ultrasound Med 1986; 5:385-389.

44. Rubin JM, Adler RS, Fowlkes JB, Carson PL. Phase cancellation: a cause of acoustical shadowing at the edges of curved surfaces in B-mode ultrasound images. Ultrasound Med Biol 1991; 17:85-95.

45. Goldstein A, Madrazo BL. Slice-thickness artifacts in grayscale ultrasound. J Clin Ultrasound 1981; 9:365-375.

46. Zdero R, Fenton PV, Bryant JT. Diagnostic ultrasound artifacts during imaging of two-body interfaces, part 2: beam thickness artifact. Ultrasonics 2002; 39:689-693.

47. Pierce G, Golding RH, Cooperberg PL. The effects of tissue velocity changes on acoustical interfaces. J Ultrasound Med 1982; 1:185-187.

48. Richman TS, Taylor KJW, Kremkau FW. Propagation speed artifact in a fatty tumor (myelolipoma): significance for tissue differential diagnosis. J Ultrasound Med 1983; 2:45-47. 


\section{Imaging Artifacts of Medical Instruments}

49. Goldstein A. Range ambiguities in real-time ultrasound. J Clin Ultrasound 1981; 9:83-90.

50. Gomberg J, Andrews G, Power J, et al. Range-gated ambiguity as a cause of artifacts in real-time echocardiography [abstract]. J Ultrasound Med 1982; 1(suppl):120.

51. Heckemann R, Seidel KJ. The sonographic appearance and contrast enhancement of puncture needles. J Clin Ultrasound 1983; 11:265-268.

52. McGahan JP. Laboratory assessment of ultrasonic needle and catheter visualization. J Ultrasound Med 1986; 5:373-377.

53. Reading CC, Charboneau JW, James EM, Hurt MR. Sonographically guided percutaneous biopsy of small $(3 \mathrm{~cm}$ or less) masses. AJR Am J Roentgenol 1988; 151:189-192.

54. Gottlieb RH, Robinette WB, Rubens DJ, Hartley DF, Fultz PJ, Violante MR. Coating agent permits improved visualization of biopsy needles during sonography. AJR Am J Roentgenol 1998; 171:1301-1302.

55. Culp WC, McCowan TC, Goertzen TC, et al. Relative ultrasonographic echogenicity of standard, dimpled, and polymeric-coated needles. J Vasc Interv Radiol 2000; 11:351358.

56. Huang J, Dupont PE, Undurti A., Triedman JK, Cleveland RO. Producing diffuse ultrasound reflections from medical instruments using a quadratic residue diffuser. Ultrasound Med Biol 2006; 32:721-727.

57. Schwartz DB, William JZ, Zagzebski J, Donovan D. The use of real-time ultrasound to enhance fetoscopic visualization. J Clin Ultrasound 1983; 11:161-164.

58. Brekhovskikh LM, Godin OA. Acoustics of Layered Media I. Berlin, Germany: Springer-Verlag; 1990:109-110. 